\title{
Characteristic Jacobi Operator on Almost Cosymplectic 3-Manifolds
}

\author{
Jun-ichi Inoguchi* \\ (Communicated by Cihan Özgür)
}

\begin{abstract}
The Ricci tensor, $\varphi$-Ricci tensor and the characteristic Jacobi operator on cosymplectic 3-manifolds are investigated.
\end{abstract}

Keywords: Almost cosymplectic manifold; characteristic Jacobi operator; Ricci tensor; Lie groups.

AMS Subject Classification (2010): Primary: 53B20 ; Secondary: 53C25; 53C30.

\section{Introduction}

The odd-dimensional real space forms provide typical examples of normal almost contact Riemannian manifolds. The almost contact structures naturally associated to the sphere $\mathbb{S}^{2 n+1}$, Euclidean space $\mathbb{E}^{2 n+1}$ and hyperbolic space $\mathbb{H}^{2 n+1}$ are classified as follows:

\begin{tabular}{|c|c|c|c|}
\hline Structure & contact metric & almost cosymplectice & almost Kenmotsu \\
\hline Normality & Sasakian & Cosymplectic & Kenmotsu \\
\hline Typical example & $\mathbb{S}^{2 n+1}$ & $\mathbb{E}^{2 n+1}$ & $\mathbb{H}^{2 n+1}$ \\
\hline
\end{tabular}

The Jacobi operator $\ell$ along the characteristic vector field $\xi$ of an almost contact Riemannian manifold plays an important role and called the characteristic Jacobi operator.

In the previous paper [14], we studied characteristic flow invariance of the characteristic Jacobi operator $\ell$ on contact Riemannian 3-manifolds, i.e., $£_{\xi} \ell=0$. Here $£_{\xi}$ denotes the Lie differentiation by $\xi$. We showed that the characteristic flow invariance of $\ell$ is weaker than the characteristic flow invariance of the Ricci operator $S$, i.e., $£_{\xi} S=0$.

A more specific subcase " $\ell=0$ " is still worth investigating. In fact, as Bang showed that the normal bundle of a Legendre submanifold in a Sasakian manifold admits a contact Riemannian structure with $\ell=0$ (See [2, Theorem 9.16]).

Contact Riemannian 3-manifolds with vanishing $\ell$ were studeid by Gouli-Andreou [28]. Kouforgiorgos and Tsichlias [37] showed that complete, simply connected, contact Riemannian 3-manifolds with vanishing $\ell$ and positive constant $|S \xi|$ are Lie groups. In our previous paper [13], we gave model spaces for the class of 3 -dimensional Lie groups equipped with left invariant contact Riemannian structure with vanishing $\ell$ and constant $|S \xi|$.

In this paper we study characteristic Jacobi operator of almost cosymplectic 3-manifolds whose characteristic Jacobi operator is invariant under the flows generated by $\xi$.

The present work has two aspects. The first aspect is to give an expository article on 3-dimensional almost cosymplectic geometry. The second aspect is to classify almost cosymplectic 3-manifolds whose characteristic Jacobi operator is invariant under the flows generated by $\xi$ under the assumption that $\xi$ is an eigenvector field of Ricci operator.

Analogues problem for almost Kenmotsu 3-manifolds will be studied in a separate publication.

Received : 30-June-2019, Accepted : 27-September-2019

* Corresponding author

Dedicated to professor Makoto Kimura on the occasion of his sixty years old

This research was partially supported by JSPS KAKENHI JP19K03461 
Conventions. In this paper we use the following definition for exterior differentiation of differential forms: Let $M$ be a manifold and $\eta$ a 1-form on $M$. Then the exterior derivative $d \eta$ is defined by

$$
2 d \eta(X, Y)=X(\eta(Y))-Y(\eta(X))-\eta([X, Y]), \quad X, Y \in \mathfrak{X}(M) .
$$

Here $\mathfrak{X}(M)$ denotes the Lie algebra of all smooth vector fields on $M$.

The exterior derivative $d \Phi$ of a 2 -form $\Phi$ is defined by

$$
d \Phi=X(\Phi(Y, Z))+Y(\Phi(Z, X))+Z(\Phi(X, Y))-\Phi([X, Y], Z)-\Phi([Y, Z], X)-\Phi([Z, X], Y) .
$$

On an oriented Riemannian manifold $(M, g), d \eta$ and $d \Phi$ are rewritten as

$$
d \eta(X, Y)=\frac{1}{2}\left(\left(\nabla_{X} \eta\right) Y-\left(\nabla_{Y} \eta\right) X\right), d \Phi(X, Y, Z)=\frac{1}{3} \mathfrak{S}_{X, Y, Z}\left(\nabla_{X} \Phi\right)(Y, Z)
$$

in terms of Levi-Civita connection $\nabla$. Here $\mathfrak{S}_{X, Y, Z}$ denotes the cyclic sum.

The codifferential $\delta \eta$ and $\delta \Phi$ are given by

$$
\delta \eta=-\operatorname{tr}(\nabla \eta), \quad(\delta \Phi) X=-\operatorname{tr}(\nabla \cdot \Phi)(\cdot, X) .
$$

\section{Preliminaries}

\section{1.}

Let $(M, g)$ be a Riemannian manifold with its Levi-Civita connection $\nabla$. Then the Riemannian curvature $R$ of $M$ is defined by

$$
R(X, Y)=\left[\nabla_{X}, \nabla_{Y}\right]-\nabla_{[X, Y]} .
$$

On a Riemannian manifold $(M, g)$, We define a curvature-like tensor field $(X, Y, Z) \longmapsto(X \wedge Y) Z$ on $M$ by

$$
(X \wedge Y) Z=g(Y, Z) X-g(Z, X) Y .
$$

A Riemannian manifold $(M, g)$ is of constant curvature $c \in \mathbb{R}$ if and only if its Riemannian curvature $R$ satisfies $R(X, Y)=c(X \wedge Y)$ for all $X, Y \in \mathfrak{X}(M)$.

As is well known, Riemannian manifolds with parallel Riemannian curvature are called locally symmetric spaces. Riemannian manifolds of constant curvature are locally symmetric.

Definition 2.1. A Riemannian manifold $(M, g)$ is said to be pseudo-symmetric if there exists a function $L$ such that

$$
R(X, Y) \cdot R=L(X \wedge Y) \cdot R
$$

holds for all $X$ and $Y \in \mathfrak{X}(M)$. In particular, a pseudo-symmetric Riemannian manifold is called a pseudosymmetric space of constant type if $L$ is constant.

In particular, pseudo-symmetric Riemannian manifolds of constant type with $L=0$ are called semi-symmetric Riemannian manifolds. Obviously, locally symmetric Riemannian manifolds are pseudo-symmetric.

The Ricci tensor field $\rho$ of $(M, g)$ is a symmetric tensor field defined by

$$
\rho(X, Y)=\operatorname{tr}(Z \longmapsto R(Z, Y) X) .
$$

The Ricci operator $S$ is a self-adjoint endomorphism field metrically equivalent to $\rho$, that is

$$
\rho(X, Y)=g(S X, Y)=g(X, S Y) .
$$

The smooth function $\mathrm{s}=\operatorname{tr} \rho=\operatorname{tr} S$ is called the scalar curvature of $(M, g)$.

A Riemannian manifold $(M, g)$ of dimension $\operatorname{dim} M \geq 3$ is said to be Einstein if $\rho=c g$ for some constant $c$. One can see that on an Einstein manifold, $\rho=(\mathrm{s} / \operatorname{dim} M) g$ and $\mathrm{s}$ is constant. Riemannian manifolds of constant curvature are Einstein.

Let $(M, g)$ be a Riemannian manifold. For a nonzero tangent vector $v \in T_{x} M$ at a point $x$, the tidal force operator $F_{v}$ associated to $v$ is a linear endomorphism on $(\mathbb{R} v)^{\perp}$ defined by $F_{v}(w):=-R(w, v) v$ for $w \perp v$ ([49, p. 219]). One can see that $F_{v}$ is self-adjoint on $(\mathbb{R} v)^{\perp}$ and has the trace $\operatorname{tr} F_{v}=-\rho(v, v)$. For a geodesic $\gamma$ in $(M, g)$, a vector field $X$ along $\gamma$ is said to be a Jacobi field along $\gamma$ if it satisfies the Jacobi equation:

$$
\nabla_{\gamma^{\prime}} \nabla_{\gamma^{\prime}} X=-F_{\gamma^{\prime}}(X) .
$$


2.2.

On a Riemannian 3-manifold $(M, g)$, the Riemannian curvature $R$ is described by the Ricci tensor field $\rho$ and corresponding Ricci operator $S$ by

$$
R(X, Y) Z=\rho(Y, Z) X-\rho(Z, X) Y+g(Y, Z) S X-g(Z, X) S Y-\frac{\mathrm{s}}{2}(X \wedge Y) Z
$$

for all vector fields $X, Y$ and $Z$ on $M$. It should be remarked that for Riemannian 3-manifolds, Einstein property is equivalent to constancy of sectional curvature.

The pseudo-symmetry is introduced as a generalization of local symmetry. In 3-dimensional Riemannian geometry, pseudo-symmetry is also a generalization of Einstein condition.

In fact, the following characterization of pseudo-symmetry is deduced.

Proposition 2.1. A Riemannian 3-manifold $\left(M^{3}, g\right)$ is a pseudo-symmetric space of constant type with $R(X, Y) \cdot R=$ $L(X \wedge Y) \cdot R$ if and only if the principal Ricci curvatures (eigenvalues of the Ricci tensor field) locally satisfy the following relations (up to numeration):

$$
\rho_{1}=\rho_{2}, \rho_{3}=2 L .
$$

Note that when $\rho_{1}=\rho_{2}=\rho_{3},\left(M^{3}, g\right)$ is Einstein, i.e., it is of constant curvature.

\section{3 .}

Let $G$ be a Lie group with a Lie algebra $\mathfrak{g}$ and a left invariant Riemannian metric $\langle\cdot, \cdot\rangle$. Then the Levi-Civita connection $\nabla$ of $(G,\langle\cdot, \cdot\rangle)$ is described by the Koszul formula:

$$
2\left\langle\nabla_{X} Y, Z\right\rangle=-\langle X,[Y, Z]\rangle+\langle Y,[Z, X]\rangle+\langle Z,[X, Y]\rangle, X, Y, Z \in \mathfrak{g} .
$$

A Lie group $G$ is said to be unimodular if its left invariant Haar measure is right invariant. Milnor gave an infinitesimal reformulation of unimodularity for 3-dimensional Lie groups [45]. We recall it briefly here.

Let $\mathfrak{g}$ be a 3-dimensional oriented Lie algebra with an inner product $\langle\cdot, \cdot\rangle$. Denote by $\times$ the vector product operation of the oriented inner product space $(\mathfrak{g},\langle\cdot, \cdot\rangle)$. The vector product operation is a skew-symmetric bilinear map $\times: \mathfrak{g} \times \mathfrak{g} \rightarrow \mathfrak{g}$ which is uniquely determined by the following conditions:

(i) $\langle X, X \times Y\rangle=\langle Y, X \times Y\rangle=0$,

(ii) $|X \times Y|^{2}=\langle X, X\rangle\langle Y, Y\rangle-\langle X, Y\rangle^{2}$,

(iii) if $X$ and $Y$ are linearly independent, then $\operatorname{det}(X, Y, X \times Y)>0$,

for all $X, Y \in \mathfrak{g}$. On the other hand, the Lie-bracket $[\cdot, \cdot]: \mathfrak{g} \times \mathfrak{g} \rightarrow \mathfrak{g}$ is a skew-symmetric bilinear map. Comparing these two operations, we get a linear endomorphism $L_{\mathfrak{g}}$ which is uniquely determined by the formula

$$
[X, Y]=L_{\mathfrak{g}}(X \times Y), \quad X, Y \in \mathfrak{g} .
$$

Now let $G$ be an oriented 3-dimensional Lie group equipped with a left invariant Riemannian metric. Then the metric induces an inner product on the Lie algebra $\mathfrak{g}$. With respect to the orientation on $\mathfrak{g}$ induced from $G$, the endomorphism field $L_{\mathfrak{g}}$ is uniquely determined. The unimodularity of $G$ is characterized as follows.

Proposition 2.2. ([45]) Let $G$ be an oriented 3-dimensional Lie group with a left invariant Riemannian metric. Then $G$ is unimodular if and only if the endomorphism $L_{\mathfrak{g}}$ is self-adjoint with respect to the metric.

Let $G$ be a Lie group with Lie algebra $\mathfrak{g}$. Denote by ad the adjoint representation of $\mathfrak{g}$,

$$
\operatorname{ad}: \mathfrak{g} \rightarrow \operatorname{End}(\mathfrak{g}) ; \operatorname{ad}(X) Y=[X, Y] .
$$

Then one can see that a map $\operatorname{tr}$ ad $: \mathfrak{g} \rightarrow \mathbb{R}$;

$$
X \longmapsto \operatorname{tr} \operatorname{ad}(X)
$$

is a Lie algebra homomorphism into the commutative Lie algebra $\mathbb{R}$. The kernel

$$
\mathfrak{u}=\{X \in \mathfrak{g} \mid \operatorname{tr} \operatorname{ad}(X)=0\}
$$

of $\operatorname{tr}$ ad is an ideal of $\mathfrak{g}$ which contains the ideal $[\mathfrak{g}, \mathfrak{g}]$.

Now we equip a left invariant Riemannian metric $\langle\cdot, \cdot\rangle$ on $G$. Denote by $\mathfrak{u}^{\perp}$ the orthogonal complement of $\mathfrak{u}$ in $\mathfrak{g}$ with respect to $\langle\cdot, \cdot\rangle$. Then the homomorphism theorem implies that $\operatorname{dim} \mathfrak{u}^{\perp}=\operatorname{dim} \mathfrak{g} / \mathfrak{u} \leq 1$.

The following criterion for unimodularity is known (see [45, p. 317]).

Lemma 2.1. A Lie group $G$ with a left invariant metric is unimodular if and only if $\mathfrak{u}=\mathfrak{g}$.

Based on this criterion, the ideal $\mathfrak{u}$ is called the unimodular kernel of $\mathfrak{g}$. In particular, for a 3-dimensional non-unimodular Lie group $G$, its unimodular kernel $\mathfrak{u}$ is commutative and of 2-dimension. 


\section{Almost contact Riemannian manifolds}

In this section we recall fundamental ingredients of almost contact Riemannian geometry. In addition we recall some curvatures of our interest. For general information on almost contact Riemannian geometry, we refer to [2].

\section{1.}

An almost contact Riemannian structure of a $(2 n+1)$-manifold $M$ is a quartet $(\varphi, \xi, \eta, g)$ of structure tensor fields which satisfies:

$$
\varphi^{2}=-\mathrm{I}+\eta \otimes \xi, \eta(\xi)=1, \quad g(\varphi X, \varphi Y)=g(X, Y)-\eta(X) \eta(Y)
$$

for all $X, Y \in \mathfrak{X}(M)$.

A $(2 n+1)$-manifold $M=(M, \varphi, \xi, \eta, g)$ equipped with an almost contact Riemannian structure is called an almost contact Riemannian manifold. The vector field $\xi$ is called the characteristic vector field of $M$. The 2-form

$$
\Phi(X, Y)=g(X, \varphi Y)
$$

is called the fundamental 2-form of $M$.

An almost contact Riemannian manifold $M$ is said to be normal if

$$
[\varphi, \varphi](X, Y)+2 d \eta(X, Y) \xi=0,
$$

for all $X, Y \in \mathfrak{X}(M)$. Here $[\varphi, \varphi]$ is the Nijenhuis torsion of $\varphi$.

Definition 3.1. Let $(M, \varphi, \xi, \eta, g)$ be an almost contact Riemannian manifold. A tangent plane $\Pi_{x}$ at $x \in M$ is said to be holomorphic if it is invariant under $\varphi_{x}$.

It is easy to see that a tangent plane $\Pi_{x}$ is holomorphic if and only if $\xi_{x}$ is orthogonal to $\Pi_{x}$. The sectional curvature $H_{x}:=K\left(\Pi_{x}\right)$ of a holomorphic plane $\Pi_{x}$ is called the holomorphic sectional curvature of $M$ at $x$.

Here we recall an auxiliary tensor field $h$ which is very useful for the study of almost contact Riemannian manifolds. The endomorphism field $h$ is defined by $h=£_{\xi} \varphi$. Here $£_{\xi}$ denotes the Lie differentiation by $\xi$. Namely

$$
h X=\frac{1}{2}\left(£_{\xi} \varphi\right) X=\frac{1}{2}\{[\xi, \varphi X]-\varphi[\xi, X]\} .
$$

3.2 .

In addition we introduce a self-adjoint endomorphism field $\ell$ on an almost contact Riemannian manifold $M$ of dimension $2 n+1 \geq 3$ by

$$
\ell(X)=R(X, \xi) \xi, \quad X \in \mathfrak{X}(M) .
$$

One can see that $\ell=-F_{\xi}$ on the distribution defined by $\eta=0$. The self-adjoint operator $\ell$ is called the characteristic Jacobi operator of $M$. Note that our $\ell$ has opposite sign to the one in [55].

Let $M$ be an almost contact Riemannian manifold. We define a tensor field $\rho^{*}$ on $M$ by (cf. [50]):

$$
\rho^{*}(X, Y):=\frac{1}{2} \operatorname{tr} R(X, \varphi Y) \varphi .
$$

One can see that $\rho^{*}(X, \xi)=0$ for all $X \in \mathfrak{X}(M)$. Next we denote by $\rho^{\varphi}$ the symmetric part of $\rho^{*}$, that is,

$$
\rho^{\varphi}(X, Y)=\frac{1}{2}\left\{\rho^{*}(X, Y)+\rho^{*}(Y, X)\right\} .
$$

We call $\rho^{\varphi}$ the $\varphi$-Ricci tensor field of $M$ [12].

Definition 3.2. An almost contact Riemannian manifold $M$ is said to be a weakly $\varphi$-Einstein manifold if

$$
\rho^{\varphi}(X, Y)=\lambda g^{\varphi}(X, Y), \quad X, Y \in \mathfrak{X}(M)
$$


for some function $\lambda$. Here the symmetric tensor field $g^{\varphi}$ is defined by

$$
g^{\varphi}(X, Y)=g(\varphi X, \varphi Y), \quad X, Y \in \mathfrak{X}(M) .
$$

When $\lambda$ is a constant, then $M$ is said to be a $\varphi$-Einstein manifold. The function $\mathrm{s}^{\varphi}=\operatorname{tr} \rho^{\varphi}$ is called the $\varphi$-scalar curvature of $M$.

Remark 3.1. An almost contact Riemannian manifold $M$ is said to be weakly $*$-Einstein if

$$
\rho^{*}(X, Y)=\lambda g(X, Y), \quad X, Y \in \mathcal{D}
$$

for some function $\lambda$. The function $\mathrm{s}^{*}=$ trace $\rho^{*}$ is called the $*$-scalar curvature of $M$. A weakly $*$-Einstein manifold of constant $*$-scalar curvature is called a $*$-Einstein manifold. Clearly $\mathrm{s}^{\varphi}=\mathrm{s}^{*}$.

To close this subsection we recall the following definition ( $c f$. [35]):

Definition 3.3. Let $M$ be an almost contact Riemannian manifold. A tensor field $P$ on $M$ of type $(1, r)$ is said to be $\eta$-parallel if

$$
g\left(\left(\nabla_{X} P\right)\left(Y_{1}, Y_{2}, \cdots, Y_{r}\right), Z\right)=0
$$

for all vector fields $X, Y_{1}, Y_{2} \cdots, Y_{r}$ and $Z$ on $M$ orthogonal to $\xi$.

The following notion was introduced by the present author (see also [36]).

Definition 3.4 ([31]). Let $M$ be an almost contact Riemannian manifold. A tensor field $P$ on $M$ of type $(1, r)$ is said to be strongly $\eta$-parallel if

$$
g\left(\left(\nabla_{X} P\right)\left(Y_{1}, Y_{2}, \cdots, Y_{r}\right), Z\right)=0
$$

for all vector fields $X, Y_{1}, Y_{2} \cdots, Y_{r}$ on $M$ and a vector field $Z$ orthogonal to $\xi$.

3.4 .

Now we turn our attention to almost cosymplectic manifolds.

Definition 3.5 ([1], [27]). An almost contact Riemannian manifold $M$ is said to be almost cosymplectic if $d \eta=0$ and $d \Phi=0$. An almost cosymplectic manifold is said to be cosymplectic if it is normal.

Note that the notion of cosymplectic manifold was introduced independently by Ogiue [47] by the name "cocomplex manifold" (see also Okumura [48]).

Remark 3.2. An almost contact Riemannian manifold $M$ is said to be semi-cosymplectic if $\delta \eta=0$ and $\delta \Phi=0$.

Olszak [50] obtained the following fundamental formula:

$$
\left(\nabla_{X} \varphi\right) Y+\left(\nabla_{\varphi X} \varphi\right)(\varphi Y)=\eta(Y) \nabla_{\varphi X} \xi .
$$

From this formula, one can deduce that

$$
\nabla_{\xi} \varphi=0, \quad \nabla_{\varphi X} \xi=-\varphi \nabla_{X} \xi, \quad \nabla_{\xi} \xi=0 .
$$

Moreover we have [19, 55]:

$$
\nabla \xi=h \varphi, h \varphi=-\varphi h, h \xi=0, \operatorname{tr} h=0, \operatorname{div} \xi=0 .
$$

The cosymplectic property is characterized as follows:

Proposition 3.1. An almost contact Riemannian manifold $M$ is cosymplectic if and only if $\varphi$ is parallel.

In particular, $\xi$ is parallel on every cosymplectic manifold. The characteristic Jacobi operator $\ell$ on an almost cosymplectic manifold $M$ satisfies (see [55, Lemma 3.1]):

$$
\nabla_{\xi} h=-h^{2} \varphi-\varphi \circ \ell, \varphi \ell \varphi-\ell=2 h^{2} .
$$

In addition we have

$$
\nabla_{\xi} h=0 \text { if and only if } \nabla_{\xi} \ell=0 .
$$

The distribution $\eta=0$ on an almost cosymplectic manifold $M$ is integrable and hence it defines a foliation $\mathcal{F}$ on $M$. This foliation is called the canonical foliation of $M$. The almost cosymplectic structure induces an almost Kähler structure on leaves. An almost cosymplectic manifold $M$ is said to be an almost cosymplectic manifold with Kähler leaves if leaves of the canonical foliation are Kähler manifolds. Clearly, if $M$ is cosymplectic then all the leaves are Kähler. 
Theorem 3.1 ([52]). An almost cosymplectic manifold has Kähler leaves if and only if

$$
\left(\nabla_{X} \varphi\right) Y=g(X, h Y)-\eta(Y) h X .
$$

Here we recall the following fundametal fact (see, e.g., [4, Theorem 3.11]).

Theorem 3.2. Let $M$ be an almost cosymplectic manifold. Then the following properties are mutually equivalent:

- $h=0$.

- The canonical foliation is totally geodesic.

- $\nabla \xi=0$.

- $\xi$ is a Killing vector field.

- $M$ is locally isomorphic to a direct product of an almost Kähler manifold and the real line.

Remark 3.3 (Compact examples). Conti and Fernández [16] constructed non-compact Einstein almost cosymplectic manifold that is non-cosymplectic. Li showed that an odd-dimensional closed manifold admits a cosymplectic structure if and only if it is diffeomorphic to a Kähler mapping torus [39]. Marrero and PadrónFernández [41] gave examples of compact cosymplectic manifolds which are not topologically equivalent to $\mathbb{C} P_{r} \times \mathbb{T}^{2 m+1}$, where $\mathbb{T}^{2 m+1}$ is a torus and $m+r=n$. Their examples are compact solvmanifolds. In particular, they showed that compact flat Riemannian 3-manifolds with non-zero first Betti number admit cosymplectic structure. The examples with first Betti number 1 are not topologically equivalent to the product manifold $\bar{M} \times \mathbb{S}^{1}$, where $\bar{M}$ is a compact surface.

\section{5.}

A complete cosymplectic manifold $M$ of constant holomorphic sectional curvature $c$ is called a cosymplectic space form. The Riemannian curvature of a cosymplectic space form has the following explicit representation $[4,40,33]$ :

$$
R(X, Y) Z=\frac{c}{4}(X \wedge Y) Z+\frac{c}{4}\{(\varphi X \wedge \varphi Y) Z+2 \Phi(X, Y) \varphi Z\}+\frac{c}{4}\{\xi \wedge(X \wedge Y) \xi\} Z .
$$

Remark 3.4. The Riemannian curvature of a Sasakian space form is given by

$$
\left.R(X, Y) Z=\frac{c+3}{4}(X \wedge Y) Z+\frac{c-1}{4}\{(\varphi X \wedge \varphi Y) Z+2 \Phi(X, Y) \varphi Z\}+\frac{c-1}{4}\{\xi \wedge(X \wedge Y) \xi\} Z\right\} .
$$

Example 3.1. Let $\bar{M}=(\bar{M}, \bar{g}, J)$ be an almost Kähler manifold. Consider a Riemannian product $M=(\bar{M} \times \mathbb{R}, g)$ with $g=\bar{g}+d t^{2}$. Then we can equip an almost cosymplectic structure on $M$ by

$$
\xi=\frac{d}{d t}, \eta=d t, \varphi\left(X, f \frac{d}{d t}\right)=(J X, 0), \quad X \in \mathfrak{X}(\bar{M}) .
$$

The almost cosymplectic manifold $M$ is cosymplectic if and only if $\bar{M}$ is Kähler. In particular when $\bar{M}$ is a complex space form, that is, a Kähler manifold of constant holomorphic sectional curvature, then $M$ is a cosymplectic manifold of constant holomorphic sectional curvature. Now let $\mathbb{C} P_{n}(c), \mathbb{C}^{n}$ and $\mathbb{C} H_{n}(c)$ be complex projective $n$-space of constant holomorphic sectional curvature $c>0$, complex Euclidean $n$-space and complex hyperbolic $n$-space of constant holomorphic sectional curvature $c<0$, respectively. Then the cosymplectic manifolds

$$
\mathbb{C} P_{n}(c) \times \mathbb{R}, \quad \mathbb{E}^{2 n+1}=\mathbb{C}^{n} \times \mathbb{R}, \quad \mathbb{C} H_{n}(c) \times \mathbb{R}
$$

are cosymplectic space forms.

Here we would like to mention another interpretation of normality of almost contact Riemannian manifolds.

Let $\left(M_{1}, \varphi_{1}, \xi_{1}, \eta_{1}, g_{1}\right)$ and $\left(M_{2}, \varphi_{2}, \xi_{2}, \eta_{2}, g_{2}\right)$ be almost contact Riemannian manifolds. Then we can introduce an almost Hermitian structure on the product manifold $M=M_{1} \times M_{2}$ by [46]:

$$
J\left(X_{1}, X_{2}\right):=\left(\varphi_{1} X_{1}-\eta_{2}\left(X_{2}\right) \xi_{1}, \varphi_{2} X_{2}+\eta_{1}\left(X_{1}\right) \xi_{2}\right) .
$$

with $g=g_{1}+g_{2}$. Morimoto [46] showed that $J$ is integrable if and only if both $M_{1}$ and $M_{2}$ are normal. If both $M_{1}$ and $M_{2}$ are Sasakian, then $M$ is Hermitian. In particular if we choose $M_{1}=\mathbb{S}^{2 p+1}$ and $M_{2}=\mathbb{S}^{2 q+1}$, then we obtain the Calabi-Eckmann manifold [3]. Since $\mathrm{H}^{2}\left(\mathbb{S}^{2 p+1} \times \mathbb{S}^{2 q+1}\right)=0$, Calabi-Eckmann manifolds can not admit any Kähler metric. Kimura [34] showed that compact complex submanifolds in the product of Sasakian 
manifolds are automatically minimal. In addition, Sierra [58] showed that complex submanifolds are totally geodesic and locally isometric to a product of Sasakian submanifolds.

On the other hand, if both $M_{1}$ and $M_{2}$ are almost cosymplectic, then $M$ is almost Kähler (Capursi [5]). In particular, $M=M_{1} \times M_{2}$ is Kähler if and only if both $M_{1}$ and $M_{2}$ are cosymplectic (Goldberg [26]).

Caprusi and Ianuş studied complex hypersurfaces in products of cosymplectic manifolds. See $[6,7,8]$.

\section{6 .}

Here we recall the notion of almost cosymplectic $(\kappa, \mu, \nu)$-space.

Definition 3.6. An almost cosymplectic manifold $M$ is said to be a generalized almost cosymplectic $(\kappa, \mu, \nu)$-space if

$$
R(X, Y) \xi=\kappa(\eta(X) Y-\eta(Y) X)+\mu(\eta(X) h Y-\eta(Y) h X)+\nu(\eta(X) \varphi h Y-\eta(Y) \varphi h X)
$$

for some smooth functions $\kappa, \mu$ and $\nu$. Generalized almost cosymplectic $(\kappa, \mu, 0)$-spaces are called generalized almost cosymplectic $(\kappa, \mu)$-spaces.

Definition 3.7. Let $M$ be a generalized almost cosymplectic $(\kappa, \mu, \nu)$-space. If both the functions $\kappa$ and $\mu$ are constants, then $M$ is called an almost cosymplectic $(\kappa, \mu, \nu)$-space. A generalized almost cosymplectic $(\kappa, \mu, \nu)$ space is said to be proper if $|d \kappa|^{2}+|d \mu|^{2}+|d \nu|^{2} \neq 0$.

Remark 3.5. Generalized almost cosymplectic $(\kappa, \mu, \nu)$-space in this paper are called almost cosymplectic $(\kappa, \mu, \nu)$ space in [57]. On the other hand, an almost cosymplectic $(\kappa, \mu, \nu)$-space in the sense of Dacko and Olszak [19] is a generalized almost cosymplectic $(\kappa, \mu, \nu)$-space in the sense of the present paper satisfying the additional condition:

$$
d \kappa \wedge \eta=0, d \mu \wedge \eta=0, \text { and } d \nu \wedge \eta=0 .
$$

Dacko and Olszak [19] showed that if the dimension of a generalized almost cosymplectic $(\kappa, \mu, \nu)$-space is greater than 3 , then $\kappa, \mu$ and $\nu$ satisfy this additional condition.

Remark 3.6. There exist almost cosymplectic $(\kappa, \mu)$-spaces which admit Einstein-Weyl structures. For this topic, we refer to Chen [10] and Matzeu [42, 43, 44].

\section{Almost cosymplectic 3-manifolds}

Hereafter we concentrate our attention to almost cosymplectic 3-manifolds.

4.1.

Let $M=(M, \varphi, \xi, \eta, g)$ be an almost contact Riemannian 3-manifold. Then the covariant derivative of $\varphi$ is given by the following Olszak formula [51]:

$$
\left(\nabla_{X} \varphi\right) Y=g\left(\varphi \nabla_{X} \xi, Y\right) \xi-\eta(Y) \varphi \nabla_{X} \xi .
$$

Olszak formula implies that an almost contact Riemannian 3-manifold is normal if and only if $\nabla \xi$ commutes with $\varphi$.

Moreover the exterior derivatives of $\eta$ and $\Phi$ of an almost contact Riemannian 3-manifold $M$ are given by

$$
d \eta=-\eta \wedge \nabla_{\xi} \eta+\frac{1}{2} \operatorname{tr}(\varphi \nabla \xi) \Phi, d \Phi=\operatorname{div} \xi \eta \wedge \Phi .
$$

These formulae imply the following fundamental fact.

Proposition 4.1. An almost contact Riemannian 3-manifold $M$ is almost cosymplectic if and only if $\nabla \xi$ is self-adjoint and $\xi$ is divergence free.

Since every almost cosymplectic manifold satisfies the anti-commutativity condition $\nabla \xi \circ \varphi=-\varphi \circ \nabla \xi$, we obtain the following characterization.

Proposition 4.2 ([50]). An almost contact Riemannian 3-manifold $M$ is cosymplectic if and only if $\xi$ is parallel. 
Here we should point out that almost cosymplectic 3-manifolds satisfy (3.1). Note that the formula (3.1) can be obtained by by inserting the formula $\nabla \xi=h \varphi$ into Olszak formula above.

Cosymplectic 3-manifolds have particular curvature properties.

Proposition 4.3. Let $M$ be a cosymplectic 3-manifold. Then the Ricci operator $S$ of $M$ has the form

$$
S=\frac{\mathrm{s}}{2}(\mathrm{I}-\eta \otimes \xi)
$$

The principal Ricci curvatures are $\mathrm{s} / 2, \mathrm{~s} / 2$ and 0 .

The Ricci operator $S$ commutes with $\varphi$. The Riemannian curvature $R$ satisfies

$$
R(X, Y) \xi=0
$$

for any vector fields $X$ and $Y$. Hence $M$ is a cosymplectic $(0,0)$-space. In particular the characteristic Jacobi operator vanishes. by

For a unit vector $X$ in $T_{x} M$ such that $\eta(X)=0$, then the sectional curvatures of planes $X \wedge \varphi X$ and $X \wedge \xi$ are given

$$
H=K(X \wedge \varphi X)=\frac{\mathrm{s}}{2}, K(X \wedge \xi)=0 .
$$

Remark 4.1. The Riemannian curvature $R$ of a cosymplectic 3-manifold has the form

$$
R(X, Y) Z=\frac{s}{2}(X \wedge Y) Z+\frac{s}{2}\{\xi \wedge(\langle(X \wedge Y) \xi\rangle)\} Z .
$$

Unfortunately in our previous papers, there are typographical errors of this formula. The first term $\frac{\mathrm{s}}{2}(X \wedge Y) Z$ is dropped in [30, Corollary 3.3] and [31, Corollary 6.1].

Note that every cosymplectic 3-manifold is semi-symmetric. The local symmetry and Einstein conditions for cosymplectic 3-manifolds are described as follows:

Corollary 4.1. The following properties of a cosymplectic 3-manifold $M$ are mutually equivalent.

- $M$ is locally symmetric.

- the scalar curvature sis constant.

- the holomorphic sectional curvature $H$ is constant.

- $M$ is locally isomorphic to a Riemannian product $\bar{M}(\bar{c}) \times \mathbb{R}$, where $\bar{M}(\bar{c})$ is a 2-dimensional Riemannian manifold of constant curvature $\bar{c}$.

Corollary 4.2. A cosymplectic 3-manifold $M$ is of constant curvature if and only if it is locally isomorphic to Euclidean 3 -space $\mathbb{E}^{3}=\mathbb{E}^{2} \times \mathbb{R}$.

\section{2.}

Let $M$ be an almost cosymplectic 3-manifold. Denote by $\mathcal{U}$ the open subset of $M$ consisting of points $x$ such that $h \neq 0$ around $x$. Next let $\mathcal{U}_{0}$ the open subset of $M$ consisting of points $x \in M$ such that $h=0$ around $x$. Since $h$ is smooth, $\mathcal{U} \cup \mathcal{U}_{0}$ is an open dense subset of $M$. So any property satisfied in $\mathcal{U} \cup \mathcal{U}_{0}$ is also satisfied in whole $M$. For any point $x \in \mathcal{U} \cup \mathcal{U}_{0}$, there exits a local orthonormal frame field $\mathcal{E}=\left\{e_{1}, e_{2}=\varphi e_{2}, e_{3}=\xi\right\}$ around $x$, where $e_{1}$ is an eigenvector field of $h$.

Lemma 4.1 (cf. [55], [53]). Let $M$ be an almost cosymplectic 3-manifold. Then there exists a local orthonormal frame field $\mathcal{E}=\left\{e_{1}, e_{2}, e_{3}\right\}$ such that

$$
h e_{1}=\lambda e_{1}, e_{2}=\varphi e_{1}, e_{3}=\xi
$$

for some locally defined smooth function $\lambda$. The Levi-Civita connection $\nabla$ is described as

$$
\begin{gathered}
\nabla_{e_{1}} e_{1}=\frac{1}{2 \lambda}\left(e_{2}(\lambda)+\sigma\left(e_{1}\right)\right), \quad \nabla_{e_{1}} e_{2}=-\frac{1}{2 \lambda}\left(e_{2}(\lambda)+\sigma\left(e_{1}\right)\right) e_{1}+\lambda \xi, \quad \nabla_{e_{1}} e_{3}=-\lambda e_{2} \\
\nabla_{e_{2}} e_{1}=-\frac{1}{2 \lambda}\left(e_{1}(\lambda)+\sigma\left(e_{2}\right)\right) e_{2}+\lambda \xi, \quad \nabla_{e_{2}} e_{2}=\frac{1}{2 \lambda}\left(e_{1}(\lambda)+\sigma\left(e_{2}\right)\right) e_{1}, \quad \nabla_{e_{2}} e_{3}=-\lambda e_{1} \\
\nabla_{e_{3}} e_{1}=\mathrm{a} e_{2}, \quad \nabla_{e_{3}} e_{2}=-\mathrm{a} e_{1}, \quad \nabla_{e_{3}} e_{3}=0
\end{gathered}
$$


where $\sigma$ is the 1-form metrically equivalen to $S \xi$, that is

$$
\sigma=g(S \xi, \cdot)=\rho(\xi, \cdot) .
$$

The commutation relations are

$$
\left[e_{1}, e_{2}\right]=-\frac{1}{2 \lambda}\left\{\left(e_{2}(\lambda)+\sigma\left(e_{1}\right)\right) e_{1}-\left(e_{1}(\lambda)+\sigma\left(e_{2}\right)\right) e_{2}\right\}, \quad\left[e_{2}, e_{3}\right]=(\mathrm{a}-\lambda) e_{1}, \quad\left[e_{3}, e_{1}\right]=(\mathrm{a}+\lambda) e_{2} .
$$

The Ricci operator $S$ is given by

$$
\begin{aligned}
S e_{1} & =\left(\frac{\mathrm{s}}{2}+\lambda^{2}-2 \mathrm{a} \lambda\right) e_{1}+\xi(\lambda) e_{2}+\sigma\left(e_{1}\right) \xi, \\
S e_{2} & =\xi(\lambda) e_{1}+\left(\frac{\mathrm{s}}{2}+\lambda^{2}+2 \mathrm{a} \lambda\right) e_{2}+\sigma\left(e_{2}\right) \xi \\
S e_{3} & =\sigma\left(e_{1}\right) e_{1}+\sigma\left(e_{2}\right) e_{2}-2 \lambda^{2} \xi .
\end{aligned}
$$

The characteristic Jacobi operator $\ell$ is given by

$$
\ell e_{1}=-\left(\lambda^{2}+2 \mathrm{a} \lambda\right) e_{1}+\xi(\lambda) e_{2}, \quad \ell e_{2}=\xi(\lambda) e_{1}-\left(\lambda^{2}-2 \mathrm{a} \lambda\right) e_{2} .
$$

The covariant derivative $\nabla_{\xi} h$ is given by

$$
\nabla_{\xi} h=\left(\frac{\xi(\lambda)}{\lambda}+2 \mathrm{a} \varphi\right) h
$$

By using the fundamental formulas

$$
\begin{aligned}
& K_{12}=K\left(e_{1} \wedge e_{2}\right)=\rho_{11}+\rho_{22}-\frac{\mathrm{s}}{2}, \\
& K_{23}=K\left(e_{2} \wedge e_{3}\right)=\rho_{22}+\rho_{23}-\frac{\mathrm{s}}{2}, \\
& K_{13}=K\left(e_{1} \wedge e_{3}\right)=\rho_{11}+\rho_{33}-\frac{\mathrm{s}}{2},
\end{aligned}
$$

we obtain the following formulas for sectional curvatures of $M$ :

$$
H=K_{12}=\frac{\mathrm{s}}{2}+2 \lambda^{2}, \quad K_{23}=-\lambda(\lambda-2 \mathrm{a}), \quad K_{13}=-\lambda(\lambda+2 \mathrm{a}) .
$$

Example 4.1 ([56]). On the Cartesian 3-space $\mathbb{R}^{3}(x, y, z)$, we define a Riemannian metric $g$ by

$$
g=d x^{2}+d y^{2}-\frac{2 y f_{1}(z)}{f_{3}(z)} d x d z-\frac{2 x f_{2}(z)}{f_{3}(z)} d y d z+\bar{f}(z) d z^{2},
$$

where $f_{1}, f_{2}$ and $f_{3}$ are arbitrary smooth functions of $z$ satisfying $f_{3} \neq 0$ and $f_{1}+f_{2} \neq 0$ on $M$. In addition we define a function $\bar{f}$ by

$$
\bar{f}(z)=\frac{y^{2} f_{1}(z)^{2}+x^{2} f_{2}(z)^{2}+1}{f_{3}(z)^{2}} .
$$

One can take an local orthonormal frame field with respect to $g$ :

$$
e_{1}=\frac{\partial}{\partial x}, \quad e_{2}=\frac{\partial}{\partial y}, \quad e_{3}=y f_{1}(z) \frac{\partial}{\partial x}+x f_{2}(z) \frac{\partial}{\partial y}+f_{3}(z) \frac{\partial}{\partial z} .
$$

We define an almost cosymplectic structure on $\mathbb{R}^{3}(x, y, z)$ by $\xi=e_{3}, \eta=g(\xi, \cdot)$ and

$$
\varphi e_{1}=e_{2}, \varphi e_{2}=-e_{1}, \varphi e_{3}=0 .
$$

Then the endomorphism field $h$ is computed as

$$
h e_{1}=\lambda e_{1}, h e_{2}=-\lambda e_{2}, h \xi=0
$$

with $\lambda=-\left(f_{1}+f_{2}\right) / 2$. One can check that this almost cosymplectic 3-manifold is a generalized almost cosymplectic $(\kappa, \mu, \nu)$-space with non-constant $\kappa, \mu$ and $\nu$.

$$
\kappa=-\frac{1}{4}\left(f_{1}+f_{2}\right)^{2}, \quad \mu=-\left(f_{1}-f_{2}\right), \quad \nu=\frac{f_{3}\left(f_{1}^{\prime}+f_{2}^{\prime}\right)}{f_{1}+f_{2}} .
$$

Here the prime denotes the differentiation by $z$. 
Remark 4.2. Erken and Murathan [24] investigated 3-dimensional almost cosymplectic $(\kappa, \mu, \nu)$-spaces satisfying $\nabla_{\xi} h=2 \mathrm{a} h \varphi$ for some function a and the gradient vector field $\operatorname{grad} \lambda$ of $\lambda$ has constant nonzero length. Murathan and his collaborators have given some examples of 3-dimensional almost cosymplectic $(\kappa, \mu, \nu)$ spaces with non-constant $\kappa, \mu$ and $\nu$ [53].

Let $M$ be a generalized almost cosymplectic $(\kappa, \mu, \nu)$-space. Take a positive constant $a$ and a smooth function $f$ such that $f \neq 0$ on $M$ and satisfies $d f \wedge \eta=0$. Then we consider a pseudo-conformal deformation

$$
\tilde{\varphi}:=\varphi, \quad \tilde{\xi}:=\frac{1}{f} \xi, \quad \tilde{\eta}:=f \eta, \quad \tilde{g}:=a^{2} g+\left(f^{2}-a^{2}\right) \eta \otimes \eta
$$

of the almost contact Riemannian structure. Then $M$ equipped with the deformed structure is a generalized almost cosymplectic $(\tilde{\kappa}, \tilde{\mu}, \tilde{\nu})$-space with

$$
\tilde{\kappa}=\frac{1}{f^{2}} \kappa, \quad \tilde{\mu}=\frac{1}{f} \mu, \quad \tilde{\nu}=\frac{1}{f^{2}}(\nu f-\xi(f)) .
$$

In particular, if we choose $a=1$ and $f=\sqrt{-\kappa}$ then $(M, \tilde{\varphi}, \tilde{\xi}, \tilde{\eta}, \tilde{g})$ is a generalized almost cosymplectic $(-1, \tilde{\mu}, 0)$-space (see [19]). Dacko and Olszak showed that every generalized almost cosymplectic $(\kappa, \mu, \nu)$-space satisfying (3.2) is pseudo-conformally deformed to a generalized almost cosymplectic $(-1, \mu, 0)$-space. For more informations on almost cosymplectic $(-1, \mu, 0)$-spaces, we refer to [20].

Remark 4.3. On an almost cosymplectic $(\kappa, \mu)$-space with constant $\kappa$ and $\mu$, we have

$$
K(X \wedge \xi)=\kappa+\lambda \mu, \quad K(X \wedge \varphi X)=-\kappa, \quad K(\varphi X \wedge \xi)=\kappa-\lambda \mu,
$$

where $X$ is an eigenvector field of $h$ corresponding to the eigenvalue $\lambda$, see $[23,4]$.

\section{Homogeneous almost cosymplectic 3-manifolds}

\section{1.}

Before investigating the characteristic flow invariance of characteristic Jacobi operators on general almost cosymplectic 3-manifolds, we study homogeneous almost cosymplectic 3-manifolds as the model cases. It should be emphasised that there exist 3-dimensional Lie groups equipped with left invariant almost cosymplectic structure which are not globally Riemannian products (see also [50]).

Definition 5.1 (cf. [55]). An almost contact Riemannian manifold $M=(M, \varphi, \xi, \eta, g)$ is said to be a homogeneous almost contact Riemannian manifold if there exists a Lie group $G$ of isometries which acts transitively on $M$ such that every element $f$ of $G$ preserves $\eta$, that is

$$
f^{*} \eta=\eta
$$

Perrone obtained the following classification.

Theorem 5.1 ([55]). Let $M$ be a simply connected homogeneous cosymplectic 3-manifold, then $M$ is either

- $M$ is one of the product Riemannian symmetric spaces

$$
\mathbb{S}^{2}(\bar{c}) \times \mathbb{R}, \quad \mathbb{H}^{2}(\bar{c}) \times \mathbb{R},
$$

where $\mathbb{S}^{2}(\bar{c})$ and $\mathbb{H}^{2}(\bar{c})$ are sphere of curvature $\bar{c}>0$ and hyperbolic plane of curvature $\bar{c}<0$ or

- $M$ itself is a Lie group $G$ equipped with left invariant almost cosymplectic structure.

\subsection{Unimodular Lie groups}

Let $G$ be a 3-dimensional unimodular Lie group with a left invariant metric $\langle\cdot, \cdot\rangle$. Then there exists an orthonormal basis $\left\{e_{1}, e_{2}, e_{3}\right\}$ of the Lie algebra $\mathfrak{g}$ such that

$$
\left[e_{1}, e_{2}\right]=c_{3} e_{3}, \quad\left[e_{2}, e_{3}\right]=c_{1} e_{1}, \quad\left[e_{3}, e_{1}\right]=c_{2} e_{2}, \quad c_{1}, c_{2}, c_{3} \in \mathbb{R} .
$$

Three-dimensional unimodular Lie groups are classified by Milnor as follows: 


\begin{tabular}{|c|c|c|}
\hline Signature of $\left(c_{1}, c_{2}, c_{3}\right)$ & Simply connected Lie group & Property \\
\hline$(+,+,+)$ & $\mathrm{SU}_{2}$ & compact and simple \\
$(-,-,+)$ or $(-,+,+)$ & $\widetilde{\mathrm{SL}}_{2} \mathbb{R}$ & non-compact and simple \\
$(+,+, 0)$ & $\widetilde{\mathrm{E}}_{2}$ & solvable \\
$(-,+, 0)$ & $\mathrm{E}_{1,1}$ & solvable \\
$(0,+, 0)$ & Heisenberg group & nilpotent \\
$(0,0,0)$ & $\left(\mathbb{R}^{3},+\right)$ & Abelian \\
\hline
\end{tabular}

To describe the Levi-Civita connection $\nabla$ of $G$, we introduce the following constants:

$$
\mu_{i}=\frac{1}{2}\left(c_{1}+c_{2}+c_{3}\right)-c_{i}
$$

Proposition 5.1. The Levi-Civita connection is given by

$$
\begin{array}{ccc}
\nabla_{e_{1}} e_{1}=0, & \nabla_{e_{1}} e_{2}=\mu_{1} e_{3}, & \nabla_{e_{1}} e_{3}=-\mu_{1} e_{2} \\
\nabla_{e_{2}} e_{1}=-\mu_{2} e_{3}, & \nabla_{e_{2}} e_{2}=0, & \nabla_{e_{2}} e_{3}=\mu_{2} e_{1} \\
\nabla_{e_{3}} e_{1}=\mu_{3} e_{2}, & \nabla_{e_{3}} e_{2}=-\mu_{3} e_{1} & \nabla_{e_{3}} e_{3}=0 .
\end{array}
$$

The Riemannian curvature $R$ is given by

$$
\begin{aligned}
& R\left(e_{1}, e_{2}\right) e_{1}=\left(\mu_{1} \mu_{2}-c_{3} \mu_{3}\right) e_{2}, \quad R\left(e_{1}, e_{2}\right) e_{2}=-\left(\mu_{1} \mu_{2}-c_{3} \mu_{3}\right) e_{1}, \\
& R\left(e_{2}, e_{3}\right) e_{2}=\left(\mu_{2} \mu_{3}-c_{1} \mu_{1}\right) e_{3}, \quad R\left(e_{2}, e_{3}\right) e_{3}=-\left(\mu_{2} \mu_{3}-c_{1} \mu_{1}\right) e_{2}, \\
& R\left(e_{1}, e_{3}\right) e_{1}=\left(\mu_{3} \mu_{1}-c_{2} \mu_{2}\right) e_{3}, \quad R\left(e_{1}, e_{3}\right) e_{3}=-\left(\mu_{3} \mu_{1}-c_{2} \mu_{2}\right) e_{1} .
\end{aligned}
$$

The basis $\left\{e_{1}, e_{2}, e_{3}\right\}$ diagonalizes the Ricci operator $S$. The principal Ricci curvatures are given by

$$
\rho_{1}=2 \mu_{2} \mu_{3}, \quad \rho_{2}=2 \mu_{1} \mu_{3}, \quad \rho_{3}=2 \mu_{1} \mu_{2} .
$$

\section{3.}

According to a result due to Perrone, simply connected 3-dimensional unimodular Lie groups equipped with left invariant almost cosymplectic structure are classified by Perrone invariant $p=\left\|£_{\xi} h\right\|-2\|h\|^{2}$ as follows:

Theorem 5.2. Let $(G, \varphi, \xi, \eta, g)$ be a simply connected 3-dimensional Lie group equipped with left invariant almost cosymplectic structure. If $G$ is unimodular, then $G$ is one of the following Lie groups;

1. If $G$ is cosympletic then $p=0$ and $G=\widetilde{\mathrm{E}}_{2}$ with flat metric or abelian group $\mathbb{R}^{3}$ equipped with Euclidean metric.

2. If $G$ is non-cosympletic, then

(a) $G=\widetilde{\mathrm{E}}_{2}$ if $p>0$.

(b) $G=$ Heisenberg group if $p=0$.

(c) $G=\mathrm{E}_{1,1}$ if $p<0$.

The Lie algebra $\mathfrak{g}$ of $G$ is generated by an orthonormal basis $\left\{e_{1}, e_{2}, e_{3}\right\}$ as in (5.1) with $c_{3}=0$. The left invariant cosymplectic structure is determined by

$$
\xi=e_{3}, \varphi e_{1}=e_{2}, \varphi e_{2}=-e_{1}, \varphi \xi=0 .
$$

Remark 5.1. Fino and Vezzoni [25] showed that Lie groups of arbitrary odd dimension which admit left invariant cosymplectic structure are flat and solvable.

Hereafter we denote by $G\left(c_{1}, c_{2}\right)$ the 3-dimensional unimodular Lie group (whose Lie algebra is determined by (5.1) with $c_{3}=0$ ) equipped with a left invariant almost cosymplectic structure. The global orthonormal frame field $\left\{e_{1}, e_{2}, e_{3}\right\}$ on $G\left(c_{1}, c_{2}\right)$ is an example of the frame field given in Lemma 4.1 with a $=\mu_{3}=\left(c_{1}+c_{2}\right) / 2$. 
Proposition 5.2. The endomorphism field $h$ of a unimodular Lie group $G$ equipped with a left invariant homogeneous almost cosymplectic structure is given by

$$
h e_{1}=-\frac{1}{2}\left(c_{1}-c_{2}\right) e_{1}, \quad h e_{2}=\frac{1}{2}\left(c_{1}-c_{2}\right) e_{2} .
$$

The sectional curvature of $G$ are given by

$$
H=K_{12}=\frac{1}{4}\left(c_{1}-c_{2}\right)^{2}, \quad K_{13}=\frac{1}{4}\left(c_{1}-c_{2}\right)\left(c_{1}+3 c_{2}\right), \quad K_{23}=-\frac{1}{4}\left(c_{1}-c_{2}\right)\left(3 c_{1}+c_{2}\right) .
$$

The principal Ricci curvatures are

$$
\rho_{1}=\frac{1}{2}\left(c_{1}^{2}-c_{2}^{2}\right), \quad \rho_{2}=-\frac{1}{2}\left(c_{1}^{2}-c_{2}^{2}\right), \quad \rho_{3}=-\frac{1}{2}\left(c_{1}-c_{2}\right)^{2} .
$$

In particular, $G\left(c_{1}, c_{2}\right)$ is scalar flat if and only if $c_{1}=c_{2}$.

This proposition implies the following classification of 3-dimensional pseudo-symmetric almost cosymplectic unimodular Lie groups (cf. [11, Proposition 11]):

Corollary 5.1 ([11]). Let $G$ be a 3-dimensional unimodular Lie group equipped with a left invariant almost cosymplectic structure. Then $G$ is pseudo-symmetric if and only if it is locally isomorphic to $\mathbb{E}^{3}, \widetilde{\mathrm{E}}_{2}, \mathrm{E}_{1,1}$ or the Heisenberg group.

More precisely one can check that

- $\rho_{1}=\rho_{2}$ if and only if $c_{2}=c_{1}$ or $c_{1}=-c_{2}$.

- $\rho_{2}=\rho_{2}$ if and only if $c_{2}=0$ or $c_{1}=c_{2}$.

- $\rho_{1}=\rho_{3}$ if and only if $c_{1}=0$ or $c_{1}=c_{2}$.

Note that pseudo-symmetry is a Riemannian notion, namely it only depends on Riemannian metric. The pseudo-symmetry of 3-dimensional Lie groups equipped with left invariant Riemannian metric was determined in [29].

Corollary 5.2. Every almost cosymplectic unimodular Lie group $G\left(c_{1}, c_{2}\right)$ is an almost cosymplectic $(\kappa, \mu)$-space with

$$
\kappa=-\frac{1}{4}\left(c_{1}-c_{2}\right)^{2}, \quad \mu=-\left(c_{1}+c_{2}\right) .
$$

The Perrone invariant of $G\left(c_{1}, c_{2}\right)$ is computed as

$$
p=-\frac{c_{1}-c_{2}}{2}\left(\sqrt{c_{1}^{2}+c_{2}^{2}}+c_{1}-c_{2}\right) .
$$

Direct computation shows that

$$
\left(£_{\xi} S\right) e_{1}=c_{2}\left(\rho_{1}-\rho_{2}\right) e_{2}, \quad\left(£_{\xi} S\right) e_{2}=c_{1}\left(\rho_{1}-\rho_{2}\right) e_{1} .
$$

Thus we obtain (see also Theorem 6.4).

Proposition 5.3. A 3-dimensional unimodular Lie group $G\left(c_{1}, c_{2}\right)$ equipped with a left invariant cosymplectic structure satisfies $£_{\xi} S=0$ if and only if $c_{2}= \pm c_{1}$. Thus the possible Lie algebras are $\mathfrak{e}_{2}, \mathfrak{e}_{1,1}$ or $\mathbb{R}^{3}$.

Proposition 5.4. The $\varphi$-Ricci tensor field of a unimodular Lie group $G\left(c_{1}, c_{2}\right)$ is given by

$$
\rho_{11}^{\varphi}=\rho_{22}^{\varphi}=H, \quad \rho_{i j}^{\varphi}=0 \text { for other } i, j
$$

Hence $G\left(c_{1}, c_{2}\right)$ is $\varphi$-Einstein. 
5.4.

The characteristic Jacobi operator $\ell$ of a unimodular Lie group $G$ is computed as

$$
\ell\left(e_{1}\right)=\ell_{1} e_{1}, \quad \ell\left(e_{2}\right)=\ell_{2} e_{2},
$$

where

$$
\ell_{1}=\frac{1}{4}\left(c_{1}-c_{2}\right)\left(c_{1}+3 c_{2}\right)=K_{13}, \quad \ell_{2}=-\frac{1}{4}\left(c_{1}-c_{2}\right)\left(3 c_{1}+c_{2}\right)=K_{23} .
$$

In particular, we have

$$
\ell_{1}+\ell_{2}=-\frac{1}{2}\left(c_{1}-c_{2}\right)^{2}, \quad \ell_{1}-\ell_{2}=\left(c_{1}-c_{2}\right)\left(c_{1}+c_{2}\right)
$$

$\ell=0$ if and only if $c_{1}=c_{2}$.

Proposition 5.5. A 3-dimensional unimodular Lie group $G\left(c_{1}, c_{2}\right)$ has vanishing characteristic Jacobi operator if and only if $G\left(c_{1}, c_{2}\right)$ is locally isometric to $\widetilde{\mathrm{E}}_{2}$ equipped with flat metric or Euclidean 3 -space $\mathbb{E}^{3}$.

Proposition 5.6. A 3-dimensional unimodular Lie group $G\left(c_{1}, c_{2}\right)$ equipped with a left invariant almost cosymplectic structure satisfies $\ell=0$ if and only if $G\left(c_{1}, c_{2}\right)$ is cosymplectic.

Direct computation shows the following fact.

Proposition 5.7. Let $G\left(c_{1}, c_{2}\right)$ be a unimodular Lie group equipped with a left invariant almost cosymplectic structure. Then $G\left(c_{1}, c_{2}\right)$ has $\eta$-parallel characteristic Jacobi operator for every $c_{1}$ and $c_{2}$.

The Lie derivative $£_{\xi} \ell$ is computed as

$$
\left(£_{\xi} \ell\right) e_{1}=c_{2}\left(\ell_{1}-\ell_{2}\right) e_{2}, \quad\left(£_{\xi} \ell\right) e_{2}=c_{1}\left(\ell_{1}-\ell_{2}\right) e_{2} .
$$

From this we obtain

Proposition 5.8. On the unimodular Lie group $G\left(c_{1}, c_{2}\right), £_{\xi} \ell=0$ holds when and only when $c_{2}= \pm c_{1}$. The possible Lie algebras are $\mathfrak{e}_{2}, \mathfrak{e}_{1,1}$ or $\mathbb{R}^{3}$.

Corollary 5.3. On the unimodular Lie group $G\left(c_{1}, c_{2}\right)$, the property $£_{\xi} \ell=0$ holds when and only when $£_{\xi} S=0$ holds.

Here we give explicit expressions of these unimodular Lie groups.

Example 5.1 (Euclidean motion group). Let us denote by $\widetilde{\mathrm{E}}_{2}$ the universal covering of the Euclidean motion group $\mathrm{E}_{2}$. Then $\widetilde{\mathrm{E}}_{2}$ is realised as $\mathbb{R}^{3}(x, y, z)$ with multiplication

$$
\left(x_{1}, y_{1}, z_{1}\right) \cdot\left(x_{2}, y_{2}, z_{2}\right)=\left(x_{1}+\left(\cos z_{1}\right) x_{2}-\left(\sin z_{1}\right) y_{2}, y_{1}+\left(\sin z_{1}\right) x_{2}+\left(\cos z_{1}\right) y_{2}, z_{1}+z_{2}\right) .
$$

For any positive real numbers $a, b, c \in \mathbb{R}$ satisfying $a \geq b$, we take a global frame field

$$
e_{1}=\frac{1}{a}\left(\cos z \frac{\partial}{\partial x}+\sin z \frac{\partial}{\partial y}\right), e_{2}=\frac{1}{b}\left(-\sin z \frac{\partial}{\partial x}+\cos z \frac{\partial}{\partial y}\right), e_{3}=\frac{1}{c} \frac{\partial}{\partial z} .
$$

Then $\left\{e_{1}, e_{2}, e_{3}\right\}$ satisfies

$$
\left[e_{1}, e_{2}\right]=0, \quad\left[e_{2}, e_{3}\right]=\frac{b c}{a} e_{1}, \quad\left[e_{3}, e_{1}\right]=\frac{b}{c a} e_{2} .
$$

The left invariant Riemannian metric $g$ determined by the condition $\left\{e_{1}, e_{2}, e_{3}\right\}$ is orthonormal with respect to it is

$$
g=a^{2} \omega^{1} \otimes \omega^{1}+b^{2} \omega^{2} \otimes \omega^{2}+c^{2} \omega^{3} \otimes \omega^{3},
$$

where

$$
\omega^{1}=\cos z d x+\sin z d y, \omega^{2}=-\sin z d x+\cos z d y, \omega^{3}=d z
$$

Let us introduce a left invariant almost contact structure by

$$
\eta=c \omega^{3}=c d z, \quad \xi=e_{3}, \varphi e_{1}=e_{2}, \varphi e_{2}=-e_{1}, \varphi e_{3}=0 .
$$

Then the resulting homogeneous almost contact Riemannian 3-manifold $\left(\widetilde{\mathrm{E}}_{2}, \varphi, \xi, \eta, g\right)$ is almost cosymplectic. The almost cosymplectic 3-manifold $\left(\widetilde{\mathrm{E}}_{2}, \varphi, \xi, \eta, g\right)$ is cosymplectic when and only when $a=b$. In such a case $g$ is flat and has the form

$$
g=a^{2}\left(d x^{2}+d y^{2}\right)+c^{2} d z^{2} .
$$

One can check that $\widetilde{\mathrm{E}}_{2}$ is pseudo-symmetric when and only when $a=b$. 
Remark 5.2. According to Patrangenaru [54], the moduli space of all left invariant Riemannian metrics on $\widetilde{\mathrm{E}}_{2}$ is parametrized as $\left\{(a, b) \in \mathbb{R}^{2} \mid a>b>0\right\} \cup\{(1,1)\}$. Every element $(a, b)$ corresponds to the metric

$$
a^{2} \omega^{1} \otimes \omega^{1}+b^{2} \omega^{2} \otimes \omega^{2}+\frac{1}{a^{2} b^{2}} \omega^{3} \otimes \omega^{3} .
$$

Example 5.2 (The Mikowski motion group). The identity component of the isometry group of Minkowski plane $\mathbb{E}^{1,1}=\left(\mathbb{R}^{2}\left(x_{1}, x_{2}\right), d x_{1} d x_{2}\right)$ is denoted by $\mathrm{E}_{1,1}$ and called the Minkowski motion group. The Minkowski motion group $\mathrm{E}_{1,1}$ is realized as $\mathbb{R}^{3}(x, y, z)$ with multiplication

$$
\left(x_{1}, y_{1}, z_{1}\right) \cdot\left(x_{2}, y_{2}, z_{2}\right)=\left(x_{1}+e^{z_{1}} x_{2}, y_{1}+e^{-z_{1}} y_{2}, z_{1}+z_{2}\right) .
$$

For any positive numbers $a, b, c$, we set

$$
e_{1}=a\left(e^{z} \frac{\partial}{\partial x}-e^{-z} \frac{\partial}{\partial y}\right), e_{2}=b\left(e^{z} \frac{\partial}{\partial x}+e^{-z} \frac{\partial}{\partial y}\right), e_{3}=c \frac{\partial}{\partial z} .
$$

Then we have

$$
\left[e_{1}, e_{2}\right]=0, \quad\left[e_{2}, e_{3}\right]=c_{1} e_{1}, \quad\left[e_{3}, e_{1}\right]=c_{2} e_{2}
$$

with

$$
c_{1}=-\frac{b c}{a}<0, \quad c_{2}=\frac{c a}{b}>0 .
$$

We equip a left invariant metric $g_{a, b, c}$ so that $\left\{e_{1}, e_{2}, e_{3}\right\}$ is orthonormal with respect to it. Then

$$
g_{a, b, c}=\frac{a^{2}+b^{2}}{4 a^{2} b^{2}}\left(e^{-2 z} d x^{2}+e^{2 z} d y^{2}\right)+\frac{d z^{2}}{c^{2}} .
$$

In particular,

$$
g_{\frac{1}{\sqrt{2}}, \frac{1}{\sqrt{2}}, 1}=e^{-2 z} d x^{2}+e^{2 z} d y^{2}+d z^{2}
$$

is the metric of the model space $\mathrm{Sol}_{3}$ of solvegeometry in the sense of Thurston.

One can check that $\left(\mathrm{E}_{1,1}, g_{a, b, c}\right)$ is pseudo-symmetric if and only if $a=b$. When $a=b$, the principal Ricci curvatures are

$$
\rho_{1}=\rho_{2}=0, \quad \rho_{3}=-\frac{4 c^{2}}{2} .
$$

Let us introduce an almost contact structure by $\xi=e_{3}, \eta=g_{a, b, c}\left(e_{3}, \cdot\right)$ and

$$
\varphi e_{1}=e_{2}, \varphi e_{2}=-e_{1}, \varphi e_{3}=0,
$$

then the Minkowski motion group equipped with this almost cosymplectic structure satisfies:

$$
\begin{gathered}
\lambda=\frac{c\left(a^{2}+b^{2}\right)}{a b} \neq 0, \quad \kappa=-\frac{\left(a^{2}+b^{2}\right)^{2} c^{2}}{4 a^{2} b^{2}}, \quad \mu=\frac{\left(a^{2}-b^{2}\right) c}{a b} . \\
H=\frac{\left(a^{2}+b^{2}\right) c^{2}}{4 a^{2} b^{2}}, \quad K_{13}=\frac{\left(a^{2}+b^{2}\right)\left(3 a^{2}-b^{2}\right) c^{2}}{4 a^{2} b^{2}}, K_{23}=\frac{\left(a^{2}+b^{2}\right)\left(a^{2}-3 b^{2}\right) c^{2}}{4 a^{2} b^{2}} .
\end{gathered}
$$

The Perrone invariant is computed as

$$
p=\frac{c^{2}\left(a^{2}+b^{2}\right)}{2 a^{2} b^{2}}\left(\sqrt{a^{4}+b^{4}}-\left(a^{2}+b^{2}\right)\right)<0 .
$$

The characteristic Jacobi operator is invariant under characteristic flow when and only when $a=b$. In particular, $\mathrm{Sol}_{3}$ equipped with compatible left invariant almost cosymplectic structure satisfies $£_{\xi} \ell=0$ but not $\ell=0$.

Remark 5.3. If we choose $\xi=e_{1}\left[\right.$ resp. $\xi=e_{2}$ ], and set $\varphi e_{2}=e_{3}, \varphi e_{3}=-e_{2}$ [resp. $\left.\varphi e_{3}=e_{1}, \varphi e_{1}=-e_{3}\right]$, then the structure is semi-cosymplectic. 
Remark 5.4. The moduli space of all left invariant Riemannian metrics on $\mathrm{E}_{1,1}$ is parametrized as $\{(A, B) \in$ $\left.\mathbb{R}^{2} \mid A>0>B \geq-A\right\}$ (see [54]). Every element $(A, B)$ corresponds to the metric

$$
\frac{A-B}{2}\left(e^{-2 z} d x^{2}+e^{2 z} d y^{2}\right)-(A+B) d x d y-\frac{1}{A B} d z^{2} .
$$

The metric $g_{a, b, c}$ is obtained as this metric under the choice

$$
A=-B=A=-B=\frac{a^{2}+b^{2}}{4 a^{2} b^{2}} .
$$

Thus the parameter $c$ is normalised as

$$
c=-A B=\frac{\left(a^{2}+b^{2}\right)^{2}}{4 a^{2} b^{2}} .
$$

Example 5.3 (The Heisenberg group). The 3 -dimensional Heisenberg group is $\mathbb{R}^{3}(x, y, z)$ together with the group structure:

$$
\left(x_{1}, y_{1}, z_{1}\right) \cdot\left(x_{2}, y_{2}, z_{2}\right):=\left(x_{1}+x_{2}, y_{1}+y_{2}, z_{1}+z_{2}+\left(x_{1} y_{2}-x_{2} y_{1}\right) / 2\right) .
$$

For any nonzero real numbers $a, b$ and $c$, we set

$$
e_{1}=b\left(2 \frac{\partial}{\partial y}+x \frac{\partial}{\partial z}\right), e_{2}=2 c \frac{\partial}{\partial z}, e_{3}=a\left(2 \frac{\partial}{\partial x}-y \frac{\partial}{\partial z}\right) .
$$

Then $\left\{e_{1}, e_{2}, e_{3}\right\}$ is a left invariant frame field satisfying the commutation relations:

$$
\left[e_{1}, e_{2}\right]=0,\left[e_{2}, e_{3}\right]=0, \quad\left[e_{3}, e_{1}\right]=c_{2} e_{2}, \quad c_{2}=\frac{a b}{c} \neq 0 .
$$

The left invariant Riemannian metric $g=g_{a, b, c}$ determined by the condition $\left\{e_{1}, e_{2}, e_{3}\right\}$ is orthonormal with respect to it is

$$
g=\frac{d x^{2}}{4 a^{2}}+\frac{d y^{2}}{4 b^{2}}+\frac{1}{4 c^{2}}\left(d z+\frac{1}{2}(y d x-x d y)\right)^{2} .
$$

One can see that the Heisenberg group equipped with this metric is pseudo-symmetric with principal Ricci curvatures $\rho_{1}=\rho_{3}=-\rho_{2}=-a b^{2} /\left(2 c^{2}\right)$. In particular the Heisenberg group equipped with the metric $g_{\frac{1}{2}, \frac{1}{2}, \frac{1}{2}}$ is the model space $\mathrm{Nil}_{3}$ of the nilgeometry in the sense of Thurston.

We introduce a left invariant almost cosymplectic structure by $\xi=e_{3}, \eta=g(\xi, \cdot)$ and

$$
\varphi e_{1}=e_{2}, \varphi e_{2}=-e_{1}, \varphi e_{3}=0 .
$$

Then the Heisenberg group equipped with this left invariant almost cosymplectic structure satisfy

$$
\begin{gathered}
h e_{1}=\frac{a b}{2 c} e_{1}, h e_{2}=-\frac{a b}{2 c} e_{2}, \quad \kappa=-\frac{a^{2} b^{2}}{4 c^{2}}, \quad \mu=-\frac{a b}{c}, \quad p=0, H=K_{12}=\frac{a^{2} b^{2}}{4 c^{2}}>0, \\
\ell_{1}=K_{13}=-\frac{3 a^{2} b^{2}}{4 c^{2}}<0, \quad \ell_{2}=K_{23}=\frac{a^{2} b^{2}}{4 c^{2}}>0 .
\end{gathered}
$$

Remark 5.5. Every left invariant Riemannian metrics on the Heisenberg group is isometric to one of the following one-parameter family of metrics ( $c f$. [54]):

$$
d x^{2}+d y^{2}+t^{2}\left(d z+\frac{1}{2}(y d x-x d y)\right)^{2}, t>0 .
$$

Remark 5.6. Dacko [18] showed that almost cosymplectic $(\kappa, 0)$-space (of arbitrary odd dimension) with $\kappa<0$ are realised as solvable Lie groups with left invariant almost cosymplectic structures. In addition those space has pseudo-parallel Ricci operator. Here we recall Dacko's construction developed in [17]. Let $G^{\prime}$ be a Lie group equipped with a left invariant almost Kähler structure. Then there exists a semi-direct product $\mathbb{R} \ltimes G^{\prime}$ equipped with a left invariant almost cosymplectic structure. 
Remark 5.7 (Compact examples). Léon [38] constructed 3-dimensional compact almost cosymplectic solvmanifolds in the following method. Let us consider the solvable Lie group

$$
G=\left\{\left(\begin{array}{ccc}
e^{k z} & 0 & x \\
0 & e^{-k z} & y \\
0 & 0 & 1
\end{array}\right) \mid x, y, z \in \mathbb{R}\right\}
$$

such that $e^{k}+e^{-k} \in \mathbb{Z} \backslash\{2\}$. One can take a right invariant vector fields

$$
e_{1}=\frac{\partial}{\partial x}, \quad e_{2}=\frac{\partial}{\partial y}, \quad e_{3}=\frac{\partial}{\partial z}-k\left(x \frac{\partial}{\partial x}-y \frac{\partial}{\partial y}\right) .
$$

The dual 1-forms are given by

$$
\omega^{1}=d x-k x d z, \omega^{2}=d y+k y d z, \omega^{3}=d z .
$$

We equip a right invariant Riemannian metric $g$ by $g=\omega^{1} \otimes \omega^{1}+\omega^{2} \otimes \omega^{2}+\omega^{3} \otimes \omega^{3}$. In addition we introduce a right invariant almost cosymplectic structure by $\eta=\omega^{3}, \xi=e_{3}$ and

$$
\varphi e_{1}=e_{2}, \varphi e_{2}=-e_{1}, \varphi e_{3}=0 .
$$

Then there exits a discrete subgroup $\Gamma$ of $G$ so that the quotient space $\Gamma \backslash G$ is a compact manifolds with induced non-cosymplectic right invariant almost cosympletic structure.

\subsection{Non-unimodular Lie groups}

Now let us consider 3-dimensional non-unimodular Lie groups equipped with left invariant almost cosymplectic structure. Here we recall Perrone's construction [55].

Let $G$ be a (simply connected) 3-dimensional non-unimodular Lie group equipped with a left invariant almost cosymplectic structure. Then one can easily check that $\xi \in \mathfrak{u}$. We take an orthonormal basis $\left\{e_{2}, e_{3}=\xi\right\}$ of $\mathfrak{u}$. Then $e_{1}=-\varphi e_{2} \in \mathfrak{u}^{\perp}$ and hence $\operatorname{ad}\left(e_{1}\right)$ preserves $\mathfrak{u}$. Express $\operatorname{ad}\left(e_{1}\right)$ as

$$
\left[e_{1}, e_{2}\right]=a_{11} e_{2}+a_{21} e_{3}, \quad\left[e_{1}, e_{3}\right]=a_{12} e_{2}+a_{22} e_{3}
$$

over $\mathfrak{u}$. The closing condition $d \eta=0$ implies that $a_{21}=0$. Next, $\nabla_{\xi} \xi=0$ implies that $a_{22}=0$. Moreover one can deduce that $\left[e_{2}, e_{3}\right]=0$ from the Jacobi identity. Note that 3-dimensional non-unimodular Lie algebras are classified by Milnor invariant $D=\operatorname{det} \operatorname{ad}\left(e_{1}\right)$.

Theorem 5.3 ([55]). Let $G$ be a 3-dimensional non-unimodular Lie group equipped with a left invariant almost cosymplectic structure. Then the Lie algebra $\mathfrak{g}=\mathfrak{g}(\gamma, \delta)$ satisfies the commutation relations

$$
\left[e_{1}, e_{2}\right]=\delta e_{2}, \quad\left[e_{2}, e_{3}\right]=0, \quad\left[e_{3}, e_{1}\right]=-\gamma e_{2},
$$

with $e_{3}=\xi, e_{1}=-\varphi e_{2} \in \mathfrak{u}^{\perp}$ and $\delta \neq 0$. In particular Milnor invariant of $\mathfrak{g}(\gamma, \delta)$ is 0 .

The Lie algebra $\mathfrak{g}=\mathfrak{g}(\gamma, \delta)$ is given explicitly by

$$
\mathfrak{g}(\gamma, \delta)=\left\{\left(\begin{array}{ccc}
(1+\delta) x & \gamma x & y \\
0 & x & z \\
0 & 0 & x
\end{array}\right) \mid x, y, z \in \mathbb{R}\right\}
$$

with basis

$$
e_{1}=\left(\begin{array}{ccc}
1+\delta & \gamma & 0 \\
0 & 1 & 0 \\
0 & 0 & 1
\end{array}\right), e_{2}=\left(\begin{array}{ccc}
0 & 0 & 1 \\
0 & 0 & 0 \\
0 & 0 & 0
\end{array}\right), e_{3}=\left(\begin{array}{lll}
0 & 0 & 0 \\
0 & 0 & 1 \\
0 & 0 & 0
\end{array}\right)
$$

The corresponding simply connected Lie group $G(\gamma, \delta)=\exp \mathfrak{g}(\gamma, \delta)$ is given by

$$
G(\gamma, \delta)=\left\{\left(\begin{array}{ccc}
e^{(1+\delta) x} & \frac{\gamma}{\delta} e^{x}\left(e^{\delta x}-1\right) & \frac{e^{x}\left((\delta y+\gamma z)\left(e^{\delta x}-1\right)-\gamma \delta x z\right)}{\delta^{2} x} \\
0 & e^{x} & z e^{x} \\
0 & 0 & e^{x}
\end{array}\right) \mid x, y, z \in \mathbb{R}\right\}
$$


The left invariant metric is expressed as $\omega^{1} \otimes \omega^{1}+\omega^{2} \otimes \omega^{2}+\eta \otimes \eta$, where

$$
\omega^{1}=d x, \omega^{2}=d y+\frac{e^{-\delta x}+\delta x-1}{\delta^{2} x^{2}}\{(\gamma y+\delta z) d x-x(\gamma d z+\delta d y)\}, \quad \eta=d z .
$$

The left invariant vector fields obtained from $e_{1}, e_{2}$ and $e_{3}$ by left translation are

$$
e_{1}=\frac{\partial}{\partial x}-\frac{e^{\delta x}\left(e^{-\delta x}+\delta x-1\right)(\delta y+\gamma z)}{\delta x\left(e^{\delta x}-1\right)} \frac{\partial}{\partial y}, \quad e_{2}=\frac{\delta x e^{\delta x}}{e^{\delta x}-1} \frac{\partial}{\partial y}, \quad e_{3}=\frac{\partial}{\partial z}-\frac{\gamma}{\delta}\left(1+\frac{x}{1-e^{-\delta x}}\right) \frac{\partial}{\partial y} .
$$

The Levi-Civita connection of $G$ is given by the following table:

Proposition 5.9. ([55])

$$
\begin{array}{ccc}
\nabla_{e_{1}} e_{1}=0, & \nabla_{e_{1}} e_{2}=-\frac{\gamma}{2} e_{3}, & \nabla_{e_{1}} e_{3}=\frac{\gamma}{2} e_{2} \\
\nabla_{e_{2}} e_{1}=-\delta e_{2}-\frac{\gamma}{2} e_{3}, & \nabla_{e_{2}} e_{2}=\delta e_{1}, & \nabla_{e_{2}} e_{3}=\frac{\gamma}{2} e_{1} \\
\nabla_{e_{3}} e_{1}=-\frac{\gamma}{2} e_{2}, & \nabla_{e_{3}} e_{2}=\frac{\gamma}{2} e_{1} & \nabla_{e_{3}} e_{3}=0 .
\end{array}
$$

The global orthonormal frame filed $\left\{e_{1}, e_{2}, e_{3}\right\}$ is an example of orthonormal frame field given in Lemma 4.1 with $\mathrm{a}=\lambda=-\gamma / 2$.

From this table, we obtain

$$
h e_{1}=-\frac{1}{2} \gamma e_{1}, h e_{2}=\frac{1}{2} \gamma e_{2} .
$$

Thus $G(\gamma, \delta)$ is cosymplectic if and only if $\gamma=0$.

The Riemannian curvature $R$ is given by

$$
\begin{aligned}
& R\left(e_{1}, e_{2}\right) e_{1}=\left(\delta^{2}-\frac{\gamma^{2}}{4}\right) e_{2}+\gamma \delta e_{3}, \quad R\left(e_{1}, e_{2}\right) e_{2}=-\left(\delta^{2}-\frac{\gamma^{2}}{4}\right) e_{1}, \\
& R\left(e_{1}, e_{3}\right) e_{1}=\gamma \delta e_{2}+\frac{3 \gamma^{2}}{4} e_{3}, \quad R\left(e_{1}, e_{3}\right) e_{3}=-\frac{3 \gamma^{2}}{4} e_{1}, \\
& R\left(e_{2}, e_{3}\right) e_{2}=-\frac{\gamma^{2}}{4} e_{3}, \quad R\left(e_{2}, e_{3}\right) e_{3}=\frac{\gamma^{2}}{4} e_{2}, \quad R\left(e_{1}, e_{2}\right) e_{3}=-\gamma \delta e_{1} .
\end{aligned}
$$

Hence

$$
H=K_{12}=-\delta^{2}+\frac{\gamma^{2}}{4}, K_{13}=-\frac{3 \gamma^{2}}{4}, K_{23}=\frac{\gamma^{2}}{4} .
$$

Thus the characteristic Jacobi operator is given by

$$
\ell\left(e_{1}\right)=K_{13} e_{1}, \quad \ell\left(e_{2}\right)=K_{23} e_{2} .
$$

Proposition 5.10. The almost cosymplectic non-unimodular Lie group $G(\gamma, \delta)$ satisfies $\ell=0$ if and only if $\gamma=0$. In this case, the structure is cosymplectic.

Thus the vanishing of $\ell$ is a too strong restriction for $G(\gamma, \delta)$.

The covariant derivatives of $\ell$ are computed as

$$
\begin{aligned}
& \left(\nabla_{e_{1}} \ell\right) e_{1}=0, \quad\left(\nabla_{e_{1}} \ell\right) e_{2}=-\frac{\gamma}{2} K_{23} e_{3}=-\frac{\gamma^{3}}{8} e_{3}, \\
& \left(\nabla_{e_{2}} \ell\right) e_{1}=-\delta\left(K_{13}-K_{23}\right) e_{2}-\frac{\gamma}{2} K_{13} e_{3}=\gamma^{2} \delta e_{2}+\frac{3 \gamma^{3}}{8} e_{3}, \\
& \left(\nabla_{e_{2}} \ell\right) e_{2}=-\delta\left(K_{13}-K_{23}\right) e_{1}=\gamma^{2} \delta e_{1} .
\end{aligned}
$$

Proposition 5.11. The characteristic Jacobi operator of the almost cosymplectic non-unimodular Lie group $G(\gamma, \delta)$ is $\eta$-parallel when and only when $\gamma=0$. In such a case $\ell=0$.

Thus unfortunately $\eta$-parallelism of $\ell$ is still a strong restriction for $G(\gamma, \delta)$.

Next we compute the Lie derivative $£_{\xi} \ell$. We obtain

$$
\left(£_{\xi} \ell\right) e_{1}=-\gamma\left(K_{13}-K_{23}\right) e_{2}=\gamma^{3} e_{2}, \quad\left(£_{\xi} \ell\right) e_{2}=0 .
$$

Proposition 5.12. The almost cosymplectic non-unimodular Lie group $G(\gamma, \delta)$ satisfies $£_{\xi} \ell=0$ if and only if $\gamma=0$ (cosymplectic). 


$$
S e_{1}=-\left(\delta^{2}+\frac{\gamma^{2}}{2}\right) e_{1}, S e_{2}=-\left(\delta^{2}-\frac{\gamma^{2}}{2}\right) e_{2}-\gamma \delta \xi, \quad S \xi=-\gamma \delta e_{2}-\frac{\gamma^{2}}{2} \xi
$$

The principal Ricci curvatures of $S$ are computed as

$$
-\frac{\gamma^{2}}{2}-\delta^{2},-\frac{\gamma^{2}}{2}-\delta^{2},-\frac{\gamma^{2}}{2}
$$

This shows that this space is pseudo-symmetric.

By using this table, the $\varphi$-Ricci tensor field is computed as

$$
\rho_{11}^{\varphi}=\rho_{22}^{\varphi}=H=\frac{\gamma^{2}}{4}-\delta^{2}, \quad \rho_{32}^{\varphi}=-\frac{1}{2} \gamma \delta .
$$

Hence $G(\gamma, \delta)$ is $\varphi$-Einstein if and only if $\gamma=0$.

The Lie group $G(0, \delta)$ is characterized as follows.

Proposition 5.13. Let $G=G(\gamma, \delta)$ be a simply connected non-unimodular Lie group corresponding to $\mathfrak{g}(\gamma, \delta)$ equipped with a left invariant almost cosymplectic structure. Then the following three conditions are mutually equivalent:

- $G$ satisfies $\gamma=0$.

- $G$ is cosymplectic.

- $\ell$ is $\eta$-parallel.

- $\ell$ is parallel.

- $\ell=0$.

- $G(\gamma, \delta)$ is $\varphi$-Einstein.

The fundamental 2-form $\Phi$ of an almost cosymplectic manifold $M$ defines a magnetic field (called the contact magnetic field) on $M$. In our previous paper [22], trajectories of contact magnetic fields on cosymplectic manifolds are investigated. In addition, we studied trajectories of magnetic fields on $\widetilde{\mathrm{E}}_{2}$ derived from the left invariant contact metric structure in [32]. Here we propose the following problem:

Problem 1. Investigate trajectories of contact magnetic fields on homogeneous almost cosymplectic 3-manifolds.

\section{Ricci curvatures of almost cosymplectic 3-manifolds}

Now we start our investigation on Ricci tensor field and related tensor fields on almost cosymplectic 3manifolds.

Locally symmetric almost cosymplectic 3-manifolds are classified as follows:

Theorem 6.1 ([55]). Let $M$ be a locally symmetric almost cosymplectic 3-manifold, then $M$ is cosymplectic and locally isomorphic to $\mathbb{S}^{2}(\bar{c}) \times \mathbb{R}, \mathbb{H}^{2}(\bar{c}) \times \mathbb{R}$ or $\mathbb{E}^{3}=\mathbb{E}^{2} \times \mathbb{R}$.

Note that this result is rephrased as

Corollary 6.1. Let $M$ be an almost cosymplectic 3-manifold. If the Ricci operator $S$ of $M$ is parallel, then $M$ is cosymplectic and locally isomorphic to $\mathbb{S}^{2}(\bar{c}) \times \mathbb{R}, \mathbb{H}^{2}(\bar{c}) \times \mathbb{R}$ or $\mathbb{E}^{3}=\mathbb{E}^{2} \times \mathbb{R}$.

This results says that local symmetry (equivalently the parallelism of $S$ ) is a very strong assumption for almost cosymplectic 3-manifolds. We are interested in more mild conditions for $S$.

One of the relaxation of local symmetry (parallelism of $S$ ) is the $\eta$-parallelism of $S$. In our previous paper [31], the present author investigated $\eta$-parallelism of $S$ of cosymplectic 3-manifolds.

Theorem 6.2 ([31]). Let $M$ be a cosymplectic 3-manifold. Then the following properties are mutually equivalent:

- The scalar curvature is constant.

- The holomorphic sectional curvature is constant.

- The Ricci operator is $\eta$-parallel.

- The Ricci operator is strongly $\eta$-parallel.

- $M$ is locally symmetric. 
Up to now, classification of almost cosymplectic 3-manifolds with $\eta$-parallel Ricci operator is still open. It is known that every cosymplectic 3 -manifold satisfies the commutativity condition $[\varphi, S]=0$.

From Lemma 4.1 one can deduce that

$$
\begin{aligned}
& S \varphi e_{1}=\varphi S e_{1} \Longleftrightarrow S \varphi e_{2}=\varphi S e_{2} \Longleftrightarrow \xi(\lambda)=0 \text { and } \mathrm{a}=0, \\
& S \varphi e_{3}=\varphi S e_{3} \Longleftrightarrow \sigma\left(e_{1}\right)=\sigma\left(e_{2}\right)=0 .
\end{aligned}
$$

Thus we obtain

Lemma 6.1 ([11]). An almost cosymplectic 3-manifold $M$ satisfies $S \varphi=\varphi S$ if and only if $M$ is cosymplectic or $M$ is non-cosympletic and satisfies

$$
\xi(\lambda)=0, \quad \mathrm{a}=0, \quad \sigma\left(e_{1}\right)=\sigma\left(e_{2}\right)=0 .
$$

By using the 2nd Bianchi identity, Cho obtained the following useful result.

Lemma 6.2 ([11]). On an almost cosymplectic 3-manifold satisfying $S \varphi=\varphi S$, the eigenvalues of the endomorphism field $h$ are constant.

As a result, almost cosymplectic 3-manifolds satisfying $S \varphi=\varphi S$ are classified as follows:

Theorem 6.3 ([11]). Let $M$ be an almost cosymplectic 3-manifold. Then $M$ satisfies $S \varphi=\varphi S$ if and only if $M$ is either cosymplectic or locally isomorphic to the Minkowski motion group $\mathrm{E}_{1,1}$ equipped with a left invariant almost cosymplectic structure described in Example 5.2.

From Proposition 5.1, one can see that unimodular Lie group $G\left(c_{1}, c_{2}\right)$ satisfies $S \varphi=\varphi S$ if and only if $c_{2}=c_{1}$ or $c_{2}=-c_{1}$. The possible Lie algebras for the former case are $\mathfrak{e}_{2}$ and $\mathbb{R}^{3}$. The latter case, the Lie algebra is $\mathfrak{e}_{1,1}$.

Motivated by the fact that every cosymplectic 3 -manifold satisfies $£_{\xi} S=0$, Cho proved the following theorem.

Theorem 6.4 ([11]). Let $M$ be an almost cosymplectic 3-manifold. Then $M$ satisfies $£_{\xi} S=0$ if and only if $M$ is either cosymplectic or locally isomorphic to the Minkowski motion group $\mathrm{E}_{1,1}$ equipped with a left invariant almost cosymplectic structure described in Example 5.2.

Generally speaking, the condition $£_{\xi} \ell=0$ is weaker that $£_{\xi} S=0$. In Section 8 we shall study this property for almost cosymplectic 3-manifolds under the assumption that $\xi$ is an eigenvector field of $S$.

Remark 6.1. Cho and Kimura investigated almost Kenmotsu 3-manifolds satisfying $£_{\xi} S=0$ [15].

\section{Generalized almost cosymplectic $(\kappa, \mu, \nu)$-spaces}

In this section we study pseudo-symmetry of $\varphi$-Ricci tensor field of 3-dimensional generalized almost cosymplectic $(\kappa, \mu, \nu)$-spaces.

In the case of contact Riemannian 3-manifolds, generalized $(\kappa, \mu, \nu)$-property is characterized by the harmonicity of the characteristic vector field. On the other hand, for almost cosymplectic 3-manifolds, generalized $(\kappa, \mu, \nu)$-property is characterized by minimality of $\xi$.

\section{1.}

Let $(M, g)$ be a Riemannian manifold with unit tangent sphere bundle $U M$. We equip the Sasaki-lift metric $g^{\mathrm{s}}$ on $U M$. Denote by $\mathfrak{X}_{1}(M)$ the space of all smooth unit vector fields on $M$. Every unit vector field $V \in \mathfrak{X}_{1}(M)$ is regarded as an immersion of $M$ into $U M$.

A unit vector field $V \in \mathfrak{X}_{1}(M)$ is said to be minimal if it is a critical point of the volume functional on $\mathfrak{X}_{1}(M)$. It is known that $V$ is a minimal unit vector field if and only if it is a minimal immersion with respect to the pull-backed metric $V^{*} g^{\text {s }}$ (see [21]).

The minimality of $\xi$ is characterized in terms of Ricci opeartor as follows.

Theorem 7.1 ([56],[27]). On an almost cosymplectic 3-manifold $M, \xi$ is a minimal unit vector field if and only if $\xi$ is an eigenvector field of $S$. 
The minimality of unit vector fields is closely related to "harmonicities" of unit vector fields.

A unit vector field $V \in \mathfrak{X}_{1}(M)$ is said to be a harmonic unit vector field if it is a critical point of the energy functional restricted to $\mathfrak{X}_{1}(M)$. On the other hand, $V$ is a harmonic map if it is a critical point of the energy functional on the space $C^{\infty}(M, U M)$ of smooth maps from $M$ into $U M$.

Perrone clarified relations between harmonicity and minimality of unit vector fields ([55, Theorem 4.3], [56, Theorem 4.2]):

Theorem 7.2. Let $M$ be an almost cosymplectic 3-manifold. Then $\xi$ is a minimal unit vector field if and only if it is a harmonic unit vector field.

The characteristic vector field $\xi$ is a harmonic map if and only if it is a harmonic unit vector field and $\xi\left(\operatorname{tr}\left(h^{2}\right)\right)=0$.

The class of almost cosymplectic 3-manifolds with minimal characteristic vector field is characterized in terms of Riemannian curvature as follows:

Theorem 7.3 ([56]). Let $M$ be an almost cosymplectic 3-manifold. If $M$ is a generalized $(\kappa, \mu, \nu)$-space, then $\xi$ is a minimal unit vector field. Conversely if $\xi$ is a minimal unit vector field, then $M$ satisfies the generalized $(\kappa, \mu, \nu)$ on an open dense subset. In such a case we have

$$
S \xi=-\left(\operatorname{tr} h^{2}\right) \xi, \quad \kappa=-\frac{1}{2}\left(\operatorname{tr} h^{2}\right) .
$$

Moreover $\operatorname{tr}\left(h^{2}\right)$ satisfies $d\left(\operatorname{tr}\left(h^{2}\right)\right) \wedge \eta=0$, that is, $X\left(\operatorname{tr}\left(h^{2}\right)\right)=0$ for any vector filed $X$ orthogonal to $\xi$. The Ricci operator has the form

$$
S=\alpha \mathrm{I}+\beta \eta \otimes \xi+\mu h+\nu \varphi h, \quad \alpha=\frac{1}{2}\left(\mathrm{~s}+\operatorname{tr}\left(h^{2}\right)\right), \quad \beta=-\frac{1}{2}\left(\mathrm{~s}+3 \operatorname{tr}\left(h^{2}\right)\right) .
$$

Let us take a local orthonormal field $\left\{e_{1}, e_{2}, e_{3}\right\}$ on a 3 -dimensional generalized almost cosymplectic $(\kappa, \mu, \nu)$ space as in Lemma 4.1, then we have

$$
S \xi=-2 \lambda^{2} \xi, \quad \kappa=-\lambda^{2}, \quad \mu=-2 \mathrm{a}, \quad \lambda \nu=\xi(\lambda) .
$$

The Ricci operator has the form

$$
\begin{aligned}
S e_{1} & =(\alpha+\lambda \mu) e_{1}+\lambda \nu e_{2}=\frac{1}{2}\left(\mathrm{~s}+2 \lambda^{2}-4 \mathrm{a} \lambda\right) e_{1}+\xi(\lambda) e_{2}, \\
S e_{2} & =\lambda \nu e_{1}+(\alpha-\lambda \mu) e_{2}=\xi(\lambda) e_{1}+\frac{1}{2}\left(\mathrm{~s}+2 \lambda^{2}+4 \mathrm{a} \lambda\right) e_{2}, \\
S e_{3} & =(\alpha+\beta) e_{3}=-2 \lambda^{2} e_{3},
\end{aligned}
$$

Remark 7.1. Carriazo and Martín-Molina [9] obtained the following curvature formula for 3-dimensional generalized almost cosymplectic $(\kappa, \mu, \nu)$-space satisfying (3.2):

$$
\begin{aligned}
R(X, Y) Z= & H(X \wedge Y) Z+(H-\kappa)\{\xi \wedge(X \wedge Y) \xi\} Z \\
& +\mu\{(h X \wedge Y) Z+(X \wedge h Y) Z\}+\nu\{(\varphi h X \wedge Y) Z+(X \wedge \varphi h Y)\}
\end{aligned}
$$

for all $X, Y$ and $Z \in \mathfrak{X}(M)$. When $M$ is a cosymplectic 3-manifold, then $\kappa=0$ and hence $H=\mathrm{s} / 2$, we retrieve the formula (4.1).

\section{2.}

As we have mentioned in Section 2, pseudo-symmetry is one of the generalisations of local symmetry. Since every cosymplectic 3 -manifold is pseudo-symmetric, we restrict our attention to non-cosymplectic case.

Now we study pseudo-symmetry of 3-dimensional generalized almost cosymplectic $(\kappa, \mu, \nu)$-spaces.

Let $M$ be a non-cosymplectic 3-dimensional almost cosymplectic $(\kappa, \mu, \nu)$-space whose characteristic vector field $\xi$ is minimal. Then by using Theorem 7.3, the characteristic polynomial $\Psi(t)=\operatorname{det}(t \mathrm{I}-S)$ for $S$ is computed as

where

$$
\Psi(t)=(t-(\alpha+\beta)) F(t),
$$

$$
F(t)=t^{2}-2 \alpha t+\alpha^{2}-\lambda^{2}\left(\mu^{2}+\nu^{2}\right) .
$$

We investigate principal Ricci curvatures. 
- Case 1: $\alpha+\beta$ is a solution to $F(t)=0$ : Direct computation shows that $F(\alpha+\beta)=0$ if and only if $\beta^{2}=\left(\mu^{2}+\nu^{2}\right)$. In this case we have $F(t)=t^{2}-2 \alpha t+\alpha^{2}-\beta^{2}$. Thus the principal Ricci curvatures are $\alpha+\beta, \alpha+\beta$ and $\alpha-\beta$. Note that

$$
\alpha+\beta=2 \kappa, \alpha-\beta=\mathrm{s}-4 \kappa=H .
$$

- Case 2: $F(t)=0$ has double roots: The discriminant $\mathcal{D}$ of $F(t)=0$ is $\mathcal{D}=4 \lambda^{2}\left(\mu^{2}+\nu^{2}\right)$. Hence $F(t)=0$ has double roots if and only if $\mu=\nu=0$ since we assume that $M$ is non-cosymplectic. Hence $M$ is an almost cosymplectic generalized $(\kappa, 0)$-space. Moreover we have a $=0$ and $\xi(\kappa)=0$. Thus $M$ satifies $S \varphi=\varphi S$. From Lemma 6.2, $\kappa$ is constant. As we have seen before $M$ is locally isomorphic to Minkowski motion group $\mathrm{E}_{1,1}$. The principal Ricci curvatures are

$$
\rho_{1}=\rho_{2}=\alpha=0, \rho_{3}=\alpha+\beta=2 \kappa=-c_{1}^{2} .
$$

Theorem 7.4. Let $M$ be a non-cosymplectic 3-dimensional generalized almost cosymplectic $(\kappa, \mu, \nu)$-space. Then $M$ is pseudo-symmetric if and only if $\mu^{2}+\nu^{2}=\left(\mathrm{s}+3 \operatorname{tr}\left(h^{2}\right)\right)^{2} / 4$ or $\mu=\nu=0$. In the latter case, $M$ is a (generalized) almost cosymplectic $(\kappa, 0)$-space and locally isomorphic to $\mathrm{E}_{1,1}$ equipped with a left invariant almost cosymplectic structure described in Example 5.2.

\section{3.}

Next we assume that $M$ is a pseudo-symmetric space of constant type.

In Case 1 of section 7.2, principal Ricci curvatures are $2 \kappa, 2 \kappa$ and $2 H$. Hence $M$ is of constant type if and only if $M$ has constant holomorphic sectional curvature $H=\alpha-\beta=\mathrm{s}-4 \kappa$.

In Case 2 of section 7.2, $\mathrm{E}_{1,1}$ is a pseudo-symmetric space of constant type.

Corollary 7.1. A 3-dimensional almost cosymplectic $(\kappa, \mu, \nu)$-space which is a pseudo-symmetric space of constant type is either (1) a cosymplectic 3-manifold, or (2) an almost cosymplectic $(\kappa, \mu, \nu)$-space satisfying $\mu^{2}+\nu^{2}=\left(\mathrm{s}+3 \operatorname{tr}\left(h^{2}\right)\right)^{2}$ with constant holomorphic sectional curvature or locally isomorphic to the Minkowski motion group $\mathrm{E}_{1,1}$ equipped with a left invariant almost cosymplectic structure described in Example 5.2.

Problem 2. Classify 3-dimensional almost cosymplectic $(\kappa, \mu, \nu)$-space satisfying $\mu^{2}+\nu^{2}=\left(\mathrm{s}+3 \operatorname{tr}\left(h^{2}\right)\right)^{2}$ with constant holomorphic sectional curvature.

7.4 .

Next let us consider Ricci $*$-tensor field of 3-dimensional generalized almost cosymplectic $(\kappa, \mu, \nu)$-spaces. Take a local orthonormal frame field $\mathcal{E}=\left\{e_{1}, e_{2}, e_{3}\right\}$ as before. Then we have

$$
R\left(e_{i}, \varphi e_{i}\right) \xi=0, \quad i=1,2,3 .
$$

Thus, for any tangent vector field $Y$, we have

$$
\left.\rho^{*}(\xi, Y)=\frac{1}{2} \sum_{i=1}^{2} g\left(R(\xi, \varphi Y) \varphi e_{i}\right), e_{i}\right)=g\left(R\left(\varphi e_{1}, e_{1}\right) \xi, \varphi Y\right)=0 .
$$

Proposition 7.1. Let $M$ be a 3-dimensional generalized almost cosymplectic $(\kappa, \mu, \nu)$-space. Then $\rho^{*}(\xi, \cdot)=0$. Hence $M$ is weakly $*$-Einstein.

Corollary 7.2. Let $M$ be a 3-dimensional generalized almost cosymplectic $(\kappa, \mu, \nu)$-space. If its $*$-scalar curvature is constant, then $M$ is $\varphi$-Einstein.

Note that on almost contact Riemannian 3-manifolds, the $*$-scalar curvature coincides with the holomorphic sectional curvature (see [12]).

\section{Characteristic flow invariant characteristic Jacobi operator}

8.1 .

In our previous paper [14] we have investigated contact Riemannian 3-manifolds satisfying $£_{\xi} \ell=0$ under the assumption that $\xi$ is an eigenvector field of $S$. In this section we study almost cosymplectic 3-manifolds satisfying $£_{\xi} \ell=0$ under the assumption that $\xi$ is an eigenvector of $S$. 
Let $M$ be an almost cosymplectic 3-manifold whose characteristic vector field is an eigenvector field of $S$. Then as we have seen before, $M$ is locally a generalized almost cosymplectic $(\kappa, \mu, \nu)$-space. In this case $S \xi=2 \kappa \xi$.

In case $M=\mathcal{U}_{0}$, then $S \xi=2 \kappa \xi$ and $£_{\xi} \ell=0$ holds on whole $M$, since $M$ is cosymplectic. Thus hereafter we assume that $\mathcal{U}$ is non-empty and take a local orthonormal frame field $\mathcal{E}=\left(e_{1}, e_{2}, e_{3}\right)$ as in Theorem 7.3.

The characteristic Jacobi operator $\ell$ is computed as

$$
\ell(X)=R(X, \xi) \xi=(\kappa I+\mu h+\nu \varphi h)(X-\eta(X) \xi)=\kappa(X-\eta(X) \xi)+\mu h X+\nu \varphi h X .
$$

Hence we get

$$
\ell\left(e_{1}\right)=(\kappa+\lambda \mu) e_{1}+\lambda \nu e_{2}, \quad \ell\left(e_{2}\right)=\lambda \nu e_{1}+(\kappa-\lambda \mu) e_{2} .
$$

Let us compute $£_{\xi} \ell$. First we observe that

$$
\left[\xi, e_{1}\right]=\left(\lambda-\frac{\mu}{2}\right) e_{2},\left[\xi, e_{2}\right]=-\left(\lambda+\frac{\mu}{2}\right) e_{1} .
$$

We put $\ell\left(e_{1}\right)=\ell_{11} e_{1}+\ell_{21} e_{2}$ and $\ell\left(e_{2}\right)=\ell_{12} e_{1}+\ell_{22} e_{2}$. Then

$$
\begin{aligned}
\left(£_{\xi} \ell\right) e_{1} & =\left[\xi, \ell\left(e_{1}\right)\right]-\ell\left[\xi, e_{1}\right]=\left[\xi, \ell_{11} e_{1}+\ell_{21} e_{2}\right]-\ell\left[\xi, e_{1}\right] \\
& =\xi\left(\ell_{11}\right) e_{1}+\xi\left(\ell_{21}\right) e_{2}+\ell_{11}\left[\xi, e_{1}\right]+\ell_{21}\left[\xi, e_{2}\right]-(\lambda+\alpha) \ell\left(e_{2}\right) \\
& =\left(\xi\left(\ell_{11}\right)-2 \lambda^{2} \nu\right) e_{1}+\left\{\xi\left(\ell_{21}\right)+2 \lambda \mu\left(\lambda-\frac{\mu}{2}\right)\right\} e_{2} \\
& =\left(\xi(\kappa+\lambda \mu)-2 \lambda^{2} \nu\right) e_{1}+\left\{\xi(\lambda \nu)+2 \lambda \mu\left(\lambda-\frac{\mu}{2}\right)\right\} e_{2},
\end{aligned}
$$

Since $\lambda \neq 0$ on $\mathcal{U},\left(£_{\xi} \ell\right) e_{1}=0$ holds if and only if

$$
\xi(\kappa+\lambda \mu)-2 \lambda^{2} \nu=0, \quad \xi(\lambda \nu)+2 \lambda \mu\left(\lambda-\frac{\mu}{2}\right)=0 .
$$

In a similar way we have $\left(£_{\xi} \ell\right) e_{2}=0$ if and only if

$$
\xi(\kappa-\lambda \mu)-\lambda \mu \nu=0, \quad \xi(\lambda \nu)-2 \lambda \mu\left(\lambda+\frac{\mu}{2}\right)=0 .
$$

Thus $£_{\xi} \ell=0$ holds if and only if $\mu=-2 \mathrm{a}=0$. In this case $\xi(\lambda)=0$ and hence $\nu=0$. Hence $M$ is a generalized almost cosymplectic $(\kappa, 0)$-space. In this case $\rho_{11}=\rho_{22}$, so $M$ is pseudo-symmetric. Moreover the orthonormal frame field $\left\{e_{1}, e_{2}, e_{3}\right\}$ satisfies

$$
\rho_{31}=2 b \lambda-e_{2}(\lambda)=\rho_{32}=2 c \lambda-e_{1}(\lambda)=0,
$$

we get $S \varphi=\varphi S$.

The condition $S \varphi=\varphi S$ implies that $\kappa$ is constant by Lemma 6.2 and hence $M$ is locally isomorphic to $\mathrm{E}_{1,1}$. Thus we arrive at our result.

Theorem 8.1. Let $M$ be an almost cosymplectic 3-manifold. Assume that $\xi$ is an eigenvector field of $S$. Then $M$ satisfies $£_{\xi} \ell=0$ if and only if $M$ is cosymplectic or a homogeneous almost cosymplectic 3-manifold, locally isomorphic to $\mathrm{E}_{1,1}$.

Up to now it is not clear that whether we can remove the assumption that $\xi$ is an eigenvector field of $S$ in the above theorem or not.

Obviously the conditions $\ell=0$ is a special case of $£_{\xi} \ell=0$. One can deduce that almost cosymplectic 3 manifolds with vanishing $\ell$ are cosymplectic from Lemma 4.1 .

Thus the Minkowski motion group $\mathrm{E}_{1,1}$ equipped with a left invariant almost cosymplectic structure provides an examples of almost cosymplectic 3-manifolds satisfying $£_{\xi} \ell=0$ but $\ell \neq 0$.

Remark 8.1. In [14, pp. 671], we have computed the Lie derivative $£_{\xi} \ell$ on a 3-dimensional non-Sasakian contact generalized $(\kappa, \mu, \nu)$-space $M$. Unfortunately the formula $£_{\xi} \ell$ given in [14, pp. 671] has typographical errors. In this opportunity we would like to give the correct formula of $£_{\xi} \ell$.

Take a local orthonormal frame field $\mathcal{E}=\left\{e_{1}, e_{2}, e_{2}=\xi\right\}$ as in [14, Lemma 3.1], then we have

$$
\begin{aligned}
\left(£_{\xi} \ell\right) e_{1} & =\left[\xi, \ell\left(e_{1}\right)\right]-\ell\left[\xi, e_{1}\right]=-2\{\xi(\lambda)(1+\lambda+2 \alpha)+\lambda \xi(\alpha)\} e_{1}+\{\xi(\xi(\lambda))-4 \alpha \lambda(1+\lambda+\alpha)\} e_{2}, \\
\left(£_{\xi} \ell\right) e_{2} & =\left[\xi, \ell\left(e_{2}\right)\right]-\ell\left[\xi, e_{2}\right]=\{\xi(\xi(\lambda))-4 \alpha \lambda(1-\lambda+\alpha)\} e_{1}+2\{\xi(\lambda)(1-\lambda+2 \alpha)+\lambda \xi(\alpha)\} e_{2} .
\end{aligned}
$$

Since we assumed that $M$ is non-Sasakian, i.e., $\lambda>0, M$ satisfies $£_{\xi} \ell=0$ if and only if $\xi(\lambda)=0$ and $\alpha=0$. From this conclusion we obtain [14, Lemma 4.4] and hence we obtain the main result [14, Theorem 4.7]. 


\section{References}

[1] Blair, D. E., The theory of quasi-Sasakian structures. Ph. D. Thesis, University of Illinois, 1966.

[2] Blair, D. E., Riemannian geometry of contact and symplectic manifolds. Progress in Mathematics. Birkhäuser Boston, Inc., Boston, second edition, 2010.

[3] Calabi, E. and Eckmann, B., A class of compact, complex manifolds which are not algebraic. Ann. Math. 58 (1953), no. 3, 494-500.

[4] Cappelletti-Montano, B., De Nicola, A., and Yudin, I., A survey on cosymplectic geometry. Rev. Math. Phys. 25 (2013), 1343002.

[5] Capursi, M., Some remarks on the product of two almost contact manifolds. An. Ştiinţ. Univ. Al. I Cuza Iaşi Secţ. I a Mat. 30 (1984), no. 1, 75-79.

[6] Capursi, M. and Ianuş, S., Complex hypersurfaces of the product of two cosymplectic manifolds I. Bull. Math. Soc. Sci. Math. R. S. Roumanie (N. S.) 28 (76) (1984), no, 4, 299-310.

[7] Capursi, M. and Ianuş, S., Complex hypersurfaces of the product of two cosymplectic manifolds II. Bull. Math. Soc. Sci. Math. R. S. Roumanie (N. S.) 29 (77) (1985), no, 3, 215-224

[8] Capursi, M. and Ianuş, S., On the scalar curvature of a complex hypersurface of the product of two cosymplectic space forms. Tensor (N.S.) 42 (1985), no. 2, 131-136.

[9] Carriazo, A. and Martín-Molina, V., Almost cosymplectic and almost Kenmotsu ( $\kappa, \mu, \nu)$-spaces. Mediterr. J. Math. 10 (2013), $1551-1571$.

[10] Chen, X., Einstein-Weyl structures on almost cosymplectic manifolds. Period. Math. Hungar., 2019, to appear.

[11] Cho, J. T., Reeb flow symmetry on almost cosymplectic three-manifolds. Bull. Korean Math. Soc. 53 (2016), no. 4, 1249-1257.

[12] Cho, J. T. and J. Inoguchi, J., On $\varphi$-Einstein contact Riemannian manifolds. Meditterr. J. Math. 7 (2010), no. 2, $143-167$.

[13] Cho, J. T. and J. Inoguchi, J., Characteristic Jacobi operator on contact Riemannian 3-manifolds. Differ. Geom. Dyn. Syst.. 17 (2015), $49-71$.

[14] Cho, J. T. and J. Inoguchi, J., Contact 3-manifolds with Reeb flow invariant characteristic Jacobi operator, An. Ştiinţ. Univ. Al. I Cuza Mat. (N. S.) 63 (2017), no. 3, 665-676.

[15] Cho, J. T. and Kimura, M., Reeb flow symmetry on almost contact three-manifolds. Differential Geom. Appl. 35 (2014), suppl., $266-273$.

[16] Conti D. and Fernández, M., Einstein almost cokähler manifolds. Math. Nachr. 289 (11-12) (2016) 1396-1407.

[17] Dacko, P., Note on left invariant almost cosymplectic structures. Period. Math. Hungar. 38 (1999), no. 3, 167-171.

[18] Dacko, P., On almost cosymplectic manifolds with the structure vector field $\xi$ belonging to the $k$-nullity distribution. Balkan J. Geom. Appl. 5 (2000), 47-60.

[19] Dacko, P. and Olszak, Z., On almost cosymplectic $(\kappa, \mu, \nu)$-space, PDEs, submanifolds and affine differential geometry, Banach Center Publ. 69 (2005), 211-220.

[20] Dacko, P. and Olszak, Z., On almost cosymplectic $(-1, \mu, 0)$-space. Cent. Europe. Sci. J. 3 (2005), no. 2, 318-330.

[21] Dragomir S. and Perrone, D., Harmonic Vector Fields: Variational Principles and Differential Geometry, Elsevier, Inc., Amsterdam, 2012.

[22] Druță-Romaniuc, S. L., Inoguchi, J., Munteanu M. I. and Nistor, A. I., Magnetic curves in cosymplectic manifolds. Rep. Math. Phys. 78 (2016), no. 1, 33-48

[23] Endo, H., Non-existence of almost cosymplectic manifolds satisfying a certain condition. Tensor (N.S.) 63 (2002), no. 3, 272-284

[24] Erken, I. K. and Murathan, C., A class of 3-dimensional almost cosymplectic manifolds. Turkish J. Math. 37 (2013), 884-894.

[25] Fino A. and Vezzoni, L., Some results on cosymplectic manifolds. Geom. Dedicata 151 (2011) 41-58.

[26] Goldberg, S. I., Totally geodesic hypersurfaces of Kaehler manifolds. Pac. J. Math. 27 (1968), no. 2, 275-281

[27] Goldberg, S. I. and Yano, K., Integrability of almost cosymplectic structures. Pac. J. Math. 31 (1969), no. 2, $373-382$.

[28] Gouli-Andreou, F., On contact metric 3-manifolds with $R(X, \xi) \xi=0$. Algebras Groups Geom. 17 (2000), 393-400.

[29] Inoguchi, J., Pseudo-symmetric Lie groups of dimension 3. Bull. Fac. Edu. Utsunomiya Univ. Sect. 2, 57 (2007), 1-5. (http://hdl. handle. net/10241/6316).

[30] Inoguchi, J., A note on almost contact Riemannian 3-manifolds. Bull. Yamagata Univ. Natur. Sci. 17 (2010), no. 1, 1-6. (http://id.nii.ac.jp/1348/00003041/).

[31] Inoguchi, J., A note on almost contact Riemannian 3-manifolds II. Bull. Korean Math. Soc. 54 (2017), no. 1, 85-97.

[32] Inoguchi, J. and Munteanu, M. I., Magnetic curves in tangent sphere bundles II. J. Math. Anal. Appl. 466 (2018) 1570-1581.

[33] Janssens D. and Vanhecke, L., Almost contact structures and curvature tensors. Kodai Math. J. 4 (1981), no. 1, 1-27.

[34] Kimura, M., Complex submanifolds of certain non-Kaehler manifolds. Kodai Math. J. 8 (1985), no. 1, 90-98.

[35] Kimura, M. and Maeda, S., On real hypersurfaces of a complex projective space. Math. Z. 202 (1989), no. 3, $299-311$.

[36] Kon, M., Invariant submanifolds in Sasakian manifolds. Math. Ann. 219 (1976), no. 3, 277-290.

[37] Koufogiorgos, T. and Tsichlias, C., Three dimensional contact metric manifolds with vanishing Jacobi operator. Beiträge Algebra Geom. 50 (2009), no. 2, 563-573

[38] de Leon, M., Compact almost contact solvmanifolds admitting neither Sasakian nor cosymplectic structures. Riv. Mat. Univ. Parma (4) 15 (1989), 105-110.

[39] Li, H., Topology of co-symplectic/co-Kähler manifolds. Asian J. Math. 12 (2008), no. 4, 527-543.

[40] Ludden, G. D., Submanifolds of cosymplectic manifolds. J. Differential Geom. 4 (1970), 237-244.

[41] Marrero J. C. and Padrón-Fernández, E., New examples of compact cosymplectic solvmanifolds. Arch. Math. (Brno) 34 (1998), no. 3, 337-345.

[42] Matzeu, P., Some examples of Einstein-Weyl structures on almost contact manifolds, Classical Quant. Grav. 17 (2000), no. 24, $5079-5087$.

[43] Matzeu, P., Almost contact Einstein-Weyl structures. Manuscripta Math. 108 (2002), no. 3, 275-288.

[44] Matzeu, P., Closed Einstein-Weyl structures on compact Sasakian and cosymplectic manifolds. Proc. Edinb. Math. Soc. (2) 54 (2011), no. 1, $149-160$.

[45] Milnor, J., Curvatures of left invariant metrics on Lie groups. Adv. Math. 21 (1976), 293-329.

[46] Morimoto, A., On normal almost contact structures. J. Math. Soc. Japan 15 (1963), no. 4, 420-436.

[47] Ogiue, K., G-structures defined by tensor fields. Kodai Math. Sem. Rep. 20 (1968), 54-75.

[48] Okumura, M., Cosymplectic hypersurfaces in Kaehlerian manifold of constant holomorphic sectional curvature. Kodai Math. Sem. Rep. 17 (1965), 63-73.

[49] O'Neill, B., Semi-Riemannian geometry with application to relativity, Academic Press, Orland, 1983.

[50] Olszak, Z., On almost cosymplectic manifolds. Kodai Math. J. 4 (1981), 239-250. 
[51] Olszak, Z., Normal almost contact metric manifolds of dimension three. Ann. Pol. Math. 47 (1986), 41-50.

[52] Olszak, Z., Almost cosymplectic manifolds with Kählerian leaves. Tensor (N. S.) 46 (1987), 117-124.

[53] Öztürk, H., Aktan, N. and Murathan, C., Almost $\alpha$-cosymplectic $(\kappa, \mu, \nu)$-spaces. preprint, 2010 (arXiv: 1007.0527v1 [math.DG]).

[54] Patrangenaru, V., Classifying 3- and 4-dimensional homogeneous Riemannian manifolds by Cartan triples. Pac. J. Math. 173 (1996), 511532.

[55] Perrone, D., Classification of homogeneous almost cosymplectic three-manifolds. Differential Geom. Appl. 30 (2012), 49-58.

[56] Perrone, D., Almost contact metric manifolds whose Reeb vector field is a harmonic section. Acta Math. Hungar. 138 (2013), no. 1-2, 102-126.

[57] Perrone, D., Minimal Reeb vector fields on almost cosymplectic manifolds. Kodai Math. J. 36 (2013), no. 1-2, 258-274.

[58] Sierra,J. M., A note on complex submanifolds of certain non-Kaehler manifolds. An. Ştiinţ. Univ. Al. I. Cuza Iaşi Secţ. I a Mat. 35 (1989), no. 2, 191-194.

\section{Affiliations}

\section{JUN-ICHI INOGUCHI}

ADDRESS: University of Tsukuba, Institute of Mathematics, 1-1-1 Tennodai, Tsukuba 305-8571, Japan.

E-MAIL: inoguchi@math.tsukuba.ac.jp ORCID ID: orcid.org/0000-0002-6584-5739 